\title{
Local improvements to reduced-order models using sensitivity analysis of the proper orthogonal decomposition
}

\author{
ALEXANDER HAY ${ }^{1} \dagger$, JEFFREY T. BORGGAARD ${ }^{1}$ \\ AND DOMINIQUE PELLETIER ${ }^{2}$ \\ ${ }^{1}$ Interdisciplinary Center for Applied Mathematics, Virginia Tech, Blacksburg, VA 24061, USA \\ ${ }_{2}^{2}$ Département de Génie Mécanique, Ecole Polytechnique de Montréal, Montréal, QC H3C3A7, Canada
}

(Received 19 June 2008 and in revised form 28 January 2009)

The proper orthogonal decomposition (POD) is the prevailing method for basis generation in the model reduction of fluids. A serious limitation of this method, however, is that it is empirical. In other words, this basis accurately represents the flow data used to generate it, but may not be accurate when applied 'off-design'. Thus, the reduced-order model may lose accuracy for flow parameters (e.g. Reynolds number, initial or boundary conditions and forcing parameters) different from those used to generate the POD basis and generally does. This paper investigates the use of sensitivity analysis in the basis selection step to partially address this limitation. We examine two strategies that use the sensitivity of the POD modes with respect to the problem parameters. Numerical experiments performed on the flow past a square cylinder over a range of Reynolds numbers demonstrate the effectiveness of these strategies. The newly derived bases allow for a more accurate representation of the flows when exploring the parameter space. Expanding the POD basis built at one state with its sensitivity leads to low-dimensional dynamical systems having attractors that approximate fairly well the attractor of the full-order Navier-Stokes equations for large parameter changes.

\section{Introduction}

A number of practical engineering problems requires the repeated simulation of unsteady fluid flows for a large number of parameter values. These problems include the control, optimization and uncertainty quantification of fluid systems. To make many of these problems tractable, reduced-order modelling has been used to minimize the simulation requirements. The use of reduced-order modelling in control and optimization has led to practical solutions for extremely challenging problems (Ito \& Ravindran 1996), such as control of low-Reynolds (Bergmann, Cordier \& Brancher 2005) and high-Reynolds number flows (Rowley \& Williams 2006), solutions to the Hamilton-Jacobi-Bellman equation arising in nonlinear feedback control (Kunisch, Volkwein \& Xie 2004) and design of materials for desired microstructure-sensitive material properties (Ganapathysubramanian \& Zabaras 2004). The development of accurate and reliable reduced-order models (ROM) is critical to the success of these solution approaches. 
Reduced-order modelling for nonlinear time-dependent problems typically consists of a mode selection strategy coupled with a model building step which generally involves Galerkin methods (Antoulas, Sorensen \& Gugercin 2001). Following Noack et al. (2003), the choices for low-dimensional global mode bases can be classified as mathematical (e.g. Noack \& Eckelmann 1994a,b), physical (e.g. Bangia et al. 1997; Morzyński, Afanasiev \& Thiele 1999) and empirical approaches. Successes, difficulties and limitations of each approach have been reported in the literature (see for example Noack et al. 2003; Bergmann et al. 2005). Empirical approaches are the most general purpose techniques in the sense that they can readily be applied to any problem regardless of the physics and the governing equations involved. They however require full-order solution data of the problem at hand on which some data processing is performed to extract global modes having some statistically relevant properties. Among them, one of the most popular approaches uses the proper orthogonal decomposition (POD) (Loève 1955) of simulation time snapshots at a given set of the parameters (see, for example, Sirovich 1987; Peterson 1989; Holmes, Lumley \& Berkooz 1996; Sirisup \& Karniadakis 2004; Antoulas 2005; Couplet, Basdevant \& Sagaut 2005). The POD and its variants are also known as Karhunen-Loève expansions (Karhunen 1946; Loève 1955), principal component analysis (PCA) (Hotelling 1933) and empirical orthogonal functions (EOF) (Lorenz 1956) among others.

This method of coupling a reduced-basis with Galerkin projection to build reducedorder models of fluid flow has developed over the past two decades as more complex simulation and control applications have emerged. The more computationally demanding the direct simulation of these problems are, the higher the need for reduced-order models.

Obviously, the accuracy of a reduced-order model crucially depends on how good the reduced basis can represent the full-order solutions. The first issue is to determine how large the truncation in the full-basis can be. A trade-off should be made between the size of the truncated basis and its capability to accurately represent the set of solutions. For turbulent flow problems, that have a wide spectrum of structures, the truncation may only be limited to preserve accuracy. To circumvent this issue, a number of specialized approaches have been proposed to model the influences of the neglected (small) structures on the reduced basis. These approaches have shown promise in managing the energy decay in the model. They are based on modifying or tuning the viscosity term in the model (Aubry et al. 1988; Sirisup \& Karniadakis 2004; Couplet et al. 2005). However, for flows where the first few modes rapidly capture all the energy up to a few tenths of $1 \%$ (as those considered in this study), these approaches are unnecessary (Deane et al. 1991).

The second issue that arises is to what extent these bases, that have been obtained from the flow data at one given set of parameters, can represent the solutions for a wide range of parameters. Indeed, the bases obtained by selection methods are shown to be optimal in a given sense (the POD basis being optimal in terms of energy representation) for the particular set of flow snapshots but are generally not well suited to represent any solution of the PDE at hand (unlike polynomial or spectral bases used in finite-element methods, for example). Thus, regardless of what truncation is used in the full basis, the reduced basis obtained may not be able to accurately represent different solutions in the parameter space potentially leading to inaccurate ROM even for the short-term dynamic simulations. This paper investigates this issue.

As a test problem, we consider the two-dimensional flow past a cylinder which has been widely used for assessing the accuracy of ROM (Deane et al. 1991; Noack et al. 2003; Galletti et al. 2004; Sirisup \& Karniadakis 2004; Couplet et al. 2005; 
Borggaard, Hay \& Pelletier 2007). The baseline configuration is set at $R e=100$ for which a ROM is built (model training). As already reported in the literature, loworder models perform well for this problem given that an accurate snapshot database from numerical simulations or experiments is available. Deane et al. (1991) have examined the ability to mimic the full simulation for Reynolds numbers beyond the values used for eigenfunction extraction. They report that the accuracy of the ROM solutions deteriorates rapidly with larger and larger changes in the parameter value. Furthermore, they show that, even though the dynamics remain low dimensional, the POD eigenspaces significantly change with Reynolds number. That is, two basis sets extracted for different Reynolds number span appreciably different subspaces. As an example, the POD modes built at $R e=100$ fail to capture on the average almost $65 \%$ of the flow energy at $R e=150$. Similarly, in the framework of flow control, Lehmann et al. (2005) have noticed that the POD model extracted from the natural attractor (uncontrolled flow) exhibits a declining ability to represent controlled flows as vortex shedding is changed. Hence, if one wishes to address flows over a range of parameter values, it is necessary to generate full-order flow data at more than a single Reynolds number. Clearly, the reduced-order modelling accuracy and robustness crucially depend on the database used to extract POD modes. As an example, Ma \& Karniadakis (2002) have successfully captured the three-dimensional bifurcation by building a basis consisting of both three- and two-dimensional POD modes extracted closely around the bifurcation point reported experimentally (note that it requires the knowledge of the bifurcation state a priori). Galletti et al. (2004) have applied the POD technique to extract a basis from a mixed database that contains snapshots at different Reynolds numbers. They have shown that the reduced-order system built from this basis captures the trends of the dynamics for Reynolds numbers not included in the database. Another approach uses generalized POD modes extracted from flow snapshots of forced transient flows (e.g. Bergmann et al. 2005; Graham, Peraire \& Tang 1999a,b). It relies on the proper choice of an ad hoc forcing term corresponding to parameter changes over the range of parameter values.

As already pointed out by Deane et al. (1991), the derived eigenfunctions cannot be identified with coherent structures at any particular parameter value. Therefore, a detailed analysis is impossible. However, these methodologies have more severe drawbacks. First, the dimension of the basis has to be substantially increased for a single ROM to predict a complete range of parameter values. This limitation can be addressed by building models at each desired state by interpolating POD basis vectors in the parameter space. Lieu, Farhat \& Lesoinne (2006) have provided an efficient way to do so by interpolating the principal angles between two POD subspaces. Morzyński et al. (2007) have interpolated the POD eigenproblem in the parameter space to yield a continuous mode interpolation procedure between two different states. Lehmann et al. (2005) have interpolated a series of very low-dimensional models to build interpolated Galerkin models specifically designed for systems where the local expansion modes geometry and dynamic model structure are preserved through parameter changes. All these approaches have been demonstrated to remove the need for a high number of modes to represent flows at various operating conditions. Secondly, the cost of the basis selection step is significantly increased since full-order data are required for several parameter values that cover the entire range to be approximated by ROM. This is the most severe limitation because the cost of the reduced-order approach rises dramatically as full-order data are expensive and demanding to obtain. Another approach is to include shift modes in the POD basis that enhance the robustness and/or the stability of the resulting Galerkin approximation. Noack et al. (2003) have 
incorporated the normalized mean-field correction in the Galerkin model. They have shown that the inclusion of this additional component allows for better prediction of the Reynolds-number dependency. Here, we propose to use sensitivity analysis in the basis selection process to enlarge the set of solutions that can be accurately represented in the parameter space which in turn allows for more accurate lowdimensional modelling.

The proposed approaches all rely on the sensitivities of the baseline POD modes (their derivatives with respect to parameters). They are derived by differentiating the eigenvalue problem that defines the set of POD modes. This yields a set of equations for the eigenvalue and eigenvector sensitivities which requires flow sensitivity data. Flow sensitivities are obtained by the Continuous Sensitivity Equation (CSE) method (see, e.g. Borggaard \& Burns 1997; Hristova et al. 2006; Ilinca, Pelletier \& Hay 2008; Pelletier et al. 2008): the partial differential equations for the flow sensitivities are derived by direct differentiation of the Navier-Stokes equations. They are then approximated and solved with similar numerical methods as for the flow equations. The appropriate methodology to derive mode sensitivities is thoroughly described. Then, we show how these sensitivities can be used to improve the capability of the POD bases to represent full-order flow solutions for a range of parameter yielding improved ROMs. We consider both the extrapolation of the baseline basis in the parameter space and its expansion using the mode sensitivities.

The paper is organized as follows. Section 2 deals with flow modelling. In $\S 2.1$, we introduce the modelling equations and their appropriate weak form for both the direct numerical simulation (DNS) and low-dimensional models. Then $\S 2.2$ presents the numerical techniques for the DNS and the description of the flows considered. In $\S 2.3$, we first present the two steps of the reduced-order modelling: the proper orthogonal decomposition and the reduced-order POD-Galerkin modelling; secondly, we examine the accuracy of ROM solutions for different values of the Reynolds number. Section 3 covers sensitivity analysis. In $\S 3.1$, we give a short presentation of the Sensitivity Equation Method (SEM) for flow sensitivity analysis. Section 3.2 describes how the POD mode sensitivities are evaluated and their accuracy is discussed in $\S 3.3$. Finally, $\S 4$ presents numerical results of improved ROMs using mode sensitivities.

\section{Flow analysis}

\subsection{Model equations}

We consider flows described by the unsteady incompressible Navier-Stokes equations. The momentum and mass conservation laws are written as

$$
\begin{aligned}
\rho \frac{\partial \boldsymbol{u}}{\partial t}+\rho(\boldsymbol{u} \cdot \nabla) \boldsymbol{u} & =\nabla \cdot \boldsymbol{\sigma}, \\
\nabla \cdot \boldsymbol{u} & =0
\end{aligned}
$$

where $\boldsymbol{u}$ is the velocity vector and $\boldsymbol{\sigma}=(-p \boldsymbol{I}+\boldsymbol{\tau}(\boldsymbol{u}))$ is the stress tensor, $\rho$ the density, $p$ the pressure and $\boldsymbol{I}$ the second-order identity tensor. For Newtonian fluids, the viscous stress tensor is given by

$$
\boldsymbol{\tau}(\boldsymbol{u})=\mu\left(\nabla \boldsymbol{u}+(\nabla \boldsymbol{u})^{T}\right)
$$

where $\mu$ is the fluid viscosity. The solution of these equations is sought on a domain $\Omega$ with a boundary $\Gamma=\Gamma_{D} \cup \Gamma_{N}$ and over times $t \in \mathscr{T}=\left(0, t_{f}\right)$. Dirichlet and homogeneous Neumann boundary conditions are imposed on boundary segments $\Gamma_{D}$ 
and $\Gamma_{N}$, respectively,

$$
\begin{aligned}
\boldsymbol{u}=\overline{\boldsymbol{u}} & \left(\Gamma_{D}\right), \\
\boldsymbol{\sigma} \cdot \boldsymbol{n}=\mathbf{0} & \left(\Gamma_{N}\right),
\end{aligned}
$$

where $\boldsymbol{n}$ is an outward unit vector normal to the boundary. The variables are initialized in time using a prescribed initial solution.

For both the DNS and the ROM simulations of this PDE, the weak forms of (2.1) and (2.2) are formed by a Galerkin projection on a set of suitable test functions. In particular, the test functions for the momentum equation satisfy the homogeneous version of the Dirichlet boundary conditions (2.4) (i.e $\boldsymbol{w} \in \boldsymbol{X}_{0}=\left\{\boldsymbol{f} \in\left[H^{1}(\Omega)\right]^{d} \mid \boldsymbol{f}=\mathbf{0}\right.$ on $\left.\Gamma_{D}\right\}$ with $d$ the dimension of the problem). Then, we seek $\boldsymbol{u} \in \boldsymbol{X}=\left[H^{1}(\Omega)\right]^{d}$ and $p \in L_{0}^{2}(\Omega)$ such that

$$
\begin{aligned}
\int_{\Omega}\left(\rho \frac{\partial \boldsymbol{u}}{\partial t}+\rho(\boldsymbol{u} \cdot \nabla) \boldsymbol{u}\right) \cdot \boldsymbol{w} \mathrm{d} \Omega & =\int_{\Omega} p(\nabla \cdot \boldsymbol{w})-\boldsymbol{\tau}: \nabla \boldsymbol{w} \mathrm{d} \Omega \quad \forall \boldsymbol{w} \in \boldsymbol{X}_{0}, \\
\int_{\Omega} q(\nabla \cdot \boldsymbol{u}) \mathrm{d} \Omega & =0 \quad \forall q \in L_{0}^{2}(\Omega) .
\end{aligned}
$$

These equations have been obtained by integrating by parts the term involving $\sigma$ as classically done

$$
\begin{aligned}
\int_{\Omega}(\nabla \cdot \sigma) \cdot \boldsymbol{w} \mathrm{d} \Omega & =\int_{\Gamma}(\boldsymbol{\sigma} \cdot \boldsymbol{w}) \cdot \boldsymbol{n} \mathrm{d} \Gamma-\int_{\Omega} \boldsymbol{\sigma}: \nabla \boldsymbol{w} \mathrm{d} \Omega \\
& =\int_{\Gamma}(\boldsymbol{\sigma} \cdot \boldsymbol{n}) \cdot \boldsymbol{w} \mathrm{d} \Gamma+\int_{\Omega} p(\nabla \cdot \boldsymbol{w})-\boldsymbol{\tau}: \nabla \boldsymbol{w} \mathrm{d} \Omega .
\end{aligned}
$$

The integrand in the boundary integral is zero on $\Gamma_{D}$ since $\boldsymbol{w} \in \boldsymbol{X}_{0}$ and also on $\Gamma_{N}$ from the homogeneous Neumann boundary condition (2.5). For the DNS, the test functions are finite-element interpolation functions that $\operatorname{span}\left[H^{1}(\Omega)\right]^{d}$ (see $\left.\S 2.2\right)$; for the ROM, the test functions are the POD modes that span the space generated by the baseline flow snapshots (see §2.3.2).

\subsection{Flow description and direct numerical simulation}

We consider here two-dimensional flows $(d=2)$ over a square cylinder classified by the Reynolds number based on the square edge length $D$ and the free stream velocity $U_{\infty}\left(R e=\rho U_{\infty} D / \mu\right)$. All Reynolds numbers examined in this study are in the range for which flows are reported to be two-dimensional, laminar and $T$-periodic in time exhibiting a Von Kármán vortex street (Okajima 1982; Sohankar, Norberg \& Davidson 1999) since $R e \in[60 ; 150]$. The baseline flow is set at $R e=100$.

As can be seen in figure 1, the computational domain $\Omega$ extends $15 D$ away from the rear cylinder edge to allow for the simulation of the convection of several vortices in the wake of the cylinder. The inflow and sides of the computational domain are located six edge lengths away. The boundary conditions on $\Gamma=\Gamma_{D} \cup \Gamma_{N}$ are set as follows. At the inlet and cylinder edges $\left(\Gamma_{D}\right)$, Dirichlet boundary conditions are applied; the no-slip condition holds on the cylinder walls $\left(\overline{\boldsymbol{u}}=[0,0]^{T}\right)$ and the free stream velocity $\left(\overline{\boldsymbol{u}}=\left[U_{\infty}, 0\right]^{T}\right)$ is prescribed at the inlet. At the sides and outlet of the computational domain $\left(\Gamma_{N}\right)$, the homogeneous Neumann boundary condition is applied as described by (2.5).

The DNS is performed by solving the discretized version of (2.6) and (2.7) using the Taylor-Hood $\left(P_{2}-P_{1}\right)$ finite-element and a mixed-formulation. Thus, 
Sources

Okajima (1982) Exp.

Davis \& Moore (1982)

Frank et al. (1990)

Norberg (1996) Exp.

Sohankar, Norberg \& Davidson (1997)

Sohankar, Norberg \& Davidson (1998)

Saha, Biswas \& Muralidhar (2003)

Present
St

$\begin{array}{cc}0.138-0.145 & - \\ 0.148-0.153 & 1.64 \\ 0.154 & 1.61 \\ 0.143 & 1.45 \\ 0.146 & 1.48 \\ 0.144-0.150 & 1.452-1.491 \\ 0.152 & 1.50 \\ 0.149 & 1.54\end{array}$

TABLE 1. Strouhal number and mean drag coefficients at $R e=100$.

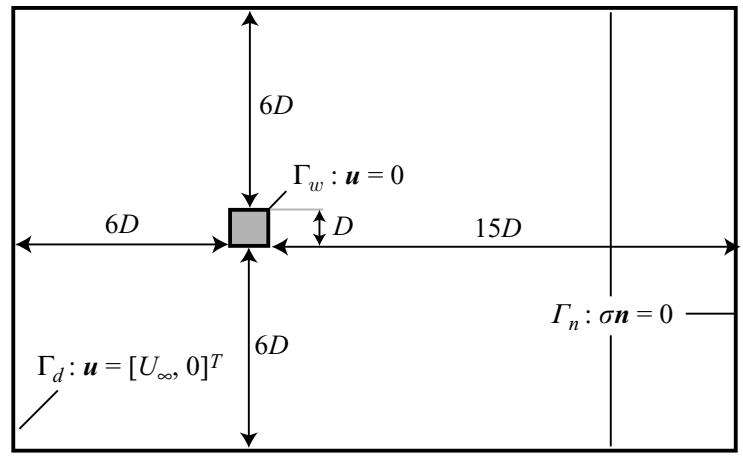

FIGURE 1. Computational configuration.

the velocity variables are discretized by six-noded quadratic interpolation functions (third-order spatial accuracy) while the pressure is discretized by piecewise linear continuous functions (second-order spatial accuracy). An implicit Crank-Nicolson time discretization of these equations (second-order temporal accuracy) leads to a system of nonlinear algebraic equations which are linearized by Newton's method and solved using a sparse direct solver.

The mesh is defined by an adaptive refinement procedure used for solving the steady-state equations for the baseline flow and sensitivity (see $\S 3$ ). Additional userdefined uniform refinement is applied in the wake region to allow for the accurate calculation of the vortex street in the unsteady calculations. The resulting mesh contains 51532 nodes and 25586 elements. Furthermore, the non-dimensional time step for the integration scheme is chosen close to 0.01 . Several refinement studies for both the space and time discretizations indicate sufficient numerical accuracy.

The DNS solution is initialized by prescribing the solution of the steady version of (2.1) and (2.2). During the first instant of the calculation, a continuous perturbation is imposed to the zero-velocity condition on the cylinder walls to force the symmetry breaking of the flow and hence speed up the onset of the vortex shedding.

The numerical results are validated by comparing the Strouhal number ( $S t=f D / U_{\infty}$, where $f$ is the vortex shedding frequency) and mean drag coefficient on the cylinder at $R e=100$ that are presently computed to those in the literature as reported in table 1 . Note that the experimental results of Norberg (1996) were originally unpublished but are reported in Sohankar et al. (1999) and suggest that $S t=0.18-3.7 / R e$ for $R e \in[50 ; 175]$ (see figure 2). Note also that the numerical results in table 1 are obtained for a set of different domain sizes and boundary conditions. 


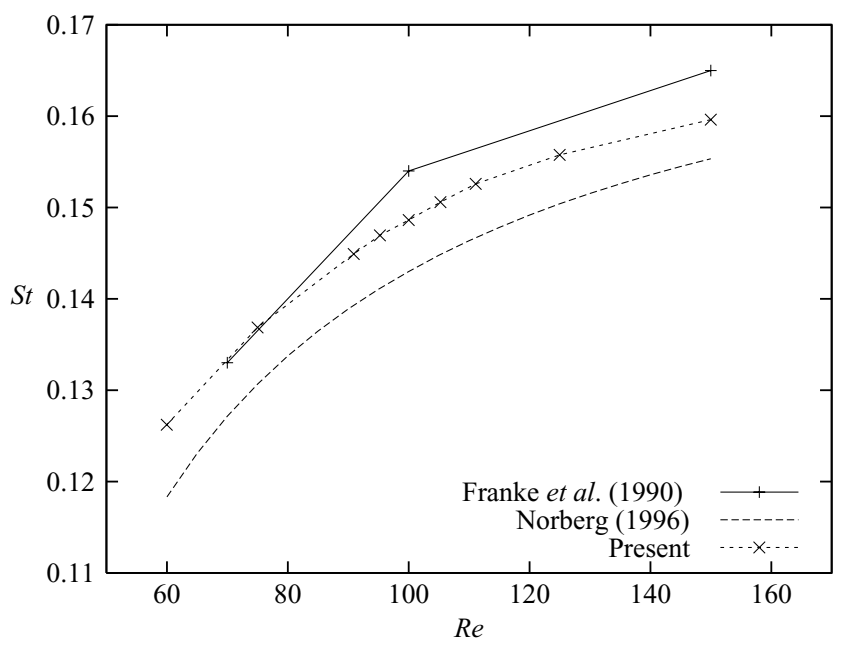

FIGURE 2. Strouhal number versus Reynolds number for the square cylinder flow.

The blockage ratios are also different but for all of them $\beta \leqslant 5 \%$. However, the present numerical results are clearly in the range of validity of what in reported in the literature.

The flows considered in what follows have a Reynolds number which ranges from 60 to 150 . Figure 2 compares the present numerical results for the Strouhal number to those by Franke, Rodi \& Schoaconung (1990) and Norberg (1996). As well established in the literature, reducing the Reynolds number results in flows exhibiting less intense vortices that are closer to the mean horizontal line so that the observed wake is thinner. It also decreases the Strouhal number which tends to zero as the flow reaches its steady regime (first supercritical Hopf bifurcation). Increasing the Reynolds number has the opposite effect up to the second supercritical Hopf bifurcation where the two-dimensional periodic solution becomes unstable to three-dimensional spanwise perturbations.

\subsection{Reduced-order modelling}

\subsubsection{The proper orthogonal decomposition}

An important step in reduced-order modelling is to find a suitable set of basis functions to represent $\boldsymbol{u}(\cdot, t) \in \boldsymbol{X}$ for $t \in(0, T)$. A natural way is to find basis elements that are well expressed over a collection of typical simulations. The present discussion will be carried out in a finite-dimensional setting since the input collection is the result of a single DNS simulation that computes discrete flow fields $\boldsymbol{u}^{h}\left(\mathbf{x}, t_{i}\right)$, at equally spaced time instants $t_{i} \in(0, T)$ for $i=1, \ldots, m$. This is referred to as the discrete POD. For a broader discussion on the proper orthogonal decomposition the interested reader is referred to Holmes et al. (1996). The developments in what follows are based on the $L^{2}$-inner product on the domain $\Omega$, denoted by $(., .)_{\Omega}$, with corresponding norm $\|.\|_{\Omega}$. Furthermore, we consider the averaging operator which is the arithmetic average so that $\boldsymbol{c}^{h}(\mathbf{x})=\left\langle\boldsymbol{u}^{h}(\mathbf{x}, t)\right\rangle=1 / m \sum_{k=1}^{m} \boldsymbol{u}^{h}\left(\mathbf{x}, t_{k}\right)$ (centring trajectory) and the POD will be applied to the data $\boldsymbol{y}_{i}^{h}(\mathbf{x})=\boldsymbol{u}^{h}\left(\mathbf{x}, t_{i}\right)-\boldsymbol{c}^{h}(\mathbf{x})$. Thus, the aim is to find a low-dimensional subspace that is the best approximation to $P=\operatorname{span}\left\{\boldsymbol{y}_{j}^{h}\right\}_{j=1}^{m}$. While fairly standard, we provide details on the notations and methodology because they will prove necessary in developing the sensitivity of the POD modes. 
Following the notation from Kunisch \& Volkwein (1999) and Fahl (2000), we assume that the input collection consists of linearly independent two-dimensional finite-element (FE) snapshots

$$
\boldsymbol{y}_{i}^{h}(\mathbf{x})=\left[\begin{array}{c}
\sum_{j=1}^{n} y_{j}^{h}\left(t_{i}\right) \varphi_{j}(\mathbf{x}) \\
\sum_{j=1}^{n} y_{n+j}^{h}\left(t_{i}\right) \varphi_{j}(\mathbf{x})
\end{array}\right]
$$

where $\left\{\varphi_{j}\right\}_{j=1}^{n}$ denote the FE interpolation functions and $n$ the number of nodes in the mesh. The snapshot data matrix $\boldsymbol{Y} \in \mathbb{R}^{2 n \times m}$ is defined as

$$
\boldsymbol{Y}=\left[\begin{array}{ccc}
y_{1}^{h}\left(t_{1}\right) & \ldots & y_{1}^{h}\left(t_{m}\right) \\
\vdots & & \vdots \\
y_{n}^{h}\left(t_{1}\right) & \ldots & y_{n}^{h}\left(t_{m}\right) \\
y_{n+1}^{h}\left(t_{1}\right) & \ldots & y_{n+1}^{h}\left(t_{m}\right) \\
\vdots & & \vdots \\
y_{2 n}^{h}\left(t_{1}\right) & \ldots & y_{2 n}^{h}\left(t_{m}\right)
\end{array}\right] .
$$

Each column of the snapshot data matrix represents a single snapshot of the input ensemble. The matrix has full column rank due to the linear independence assumption on the FE snapshots. Similarly, any two-dimensional vector function is discretized as

$$
\boldsymbol{\phi}(\mathbf{x})=\left[\begin{array}{c}
\sum_{j=1}^{n} \phi_{j} \varphi_{j}(\mathbf{x}) \\
\sum_{j=1}^{n} \phi_{n+j} \varphi_{j}(\mathbf{x})
\end{array}\right]
$$

so that the $\boldsymbol{L}^{2}$-inner product is computed by the $\boldsymbol{M}$-inner product: $(\boldsymbol{\psi}, \boldsymbol{\phi})_{\Omega}=\boldsymbol{\psi}^{T} \boldsymbol{M} \boldsymbol{\phi}$ where $\boldsymbol{M} \in \mathbb{R}^{2 n \times 2 n}$ is the symmetric positive definite (generally sparse) FE mass matrix. Note that we are using $\phi$ for both the FE approximation as well as the coefficients of its expansion in the basis $\varphi$. As noted by Kunisch \& Volkwein (1999) and Fahl (2000), using the Cholesky factorization of the mass matrix, $\boldsymbol{M}=\boldsymbol{L L}^{T}$, the $\boldsymbol{M}$-inner product is reduced to a standard Euclidean inner product of the vectors that are left multiplied by $\boldsymbol{L}^{T}$

$$
(\boldsymbol{\psi}, \boldsymbol{\phi})_{\Omega}=\left(\boldsymbol{L}^{T} \boldsymbol{\psi}\right)^{T}\left(\boldsymbol{L}^{T} \boldsymbol{\phi}\right) .
$$

Thus, we define the weighted snapshot data matrix

$$
\boldsymbol{A}=\boldsymbol{L}^{T} \boldsymbol{Y} \in \mathbb{R}^{2 n \times m} .
$$

The so-called method of snapshots introduced by Sirovich (1987) expresses the POD vectors as a linear combination of the snapshots which leads to the following eigenproblem:

$$
\boldsymbol{A}^{T} \boldsymbol{A} \boldsymbol{V}=\boldsymbol{V} \boldsymbol{\Lambda} \text { with } \boldsymbol{\Phi}=\boldsymbol{L}^{-T} \boldsymbol{A} \boldsymbol{V} \boldsymbol{\Lambda}^{-1 / 2}=\boldsymbol{Y} \boldsymbol{V} \boldsymbol{\Lambda}^{-1 / 2},
$$

where the matrix $\boldsymbol{\Phi} \in \mathbb{R}^{2 n \times m}$ collects the FE coefficients of the discrete POD basis functions and $\boldsymbol{\Lambda}=\operatorname{diag}\left(\lambda_{1}, \ldots, \lambda_{m}\right)$. Each column of $\boldsymbol{\Phi}$ represents a single POD vector $\boldsymbol{\phi}_{j}$ and they are ordered such that $\lambda_{i} \geqslant \lambda_{i+1}$. Due to the assumptions on the FE snapshots, the matrix $\boldsymbol{A}^{T} \boldsymbol{A} \in \mathbb{R}^{m \times m}$ is symmetric positive definite so that the spectral theorem guarantees a full orthogonal set of real eigenvectors associated to strictly positive real eigenvalues. From the physical point of view, $\lambda_{i}$ measures the average kinetic energy of the data captured by $\phi_{i}$ over $(0, T)$.

The POD basis of dimension $q$ is defined as a set of $q$ dominant eigenfunctions. Thus, the subspace $P^{q}=\operatorname{span}\left\{\boldsymbol{\phi}_{j}\right\}_{j=1}^{q}$ is the best approximating subspace of dimension 


\begin{tabular}{lcccccccccc}
\hline$q$ & 1 & 2 & 3 & 4 & 5 & 6 & 7 & 8 & 9 & 10 \\
$E_{q}(R e=150)$ & 0.5058 & 0.9570 & 0.9686 & 0.9785 & 0.9882 & 0.9977 & 0.9985 & 0.9992 & 0.9995 & 0.9998 \\
$E_{q}(R e=100)$ & 0.4848 & 0.9515 & 0.9668 & 0.9819 & 0.9902 & 0.9985 & 0.9991 & 0.9997 & 0.9998 & 0.9999 \\
$E_{q}(R e=60)$ & 0.4868 & 0.9523 & 0.9722 & 0.9920 & 0.9957 & 0.9994 & 0.9997 & 0.9999 & 0.9999 & 0.9999
\end{tabular}

TABLE 2. Effectiveness of the POD for different Reynolds numbers.

$q$ to $P$ in terms of kinetic energy representation. One measure of the effectiveness of the POD is related to the rate of decay of the eigenvalues and measured as

$$
E_{q}=\frac{\sum_{j=1}^{q} \lambda_{j}}{\sum_{j=1}^{m} \lambda_{j}}
$$

\subsubsection{Reduced-order POD-Galerkin modelling}

From $\S 2.3 .1$, the approximation $\boldsymbol{u}^{r}$ of order $q$ of $\boldsymbol{u}$ is

$$
\boldsymbol{u}^{r}(\mathbf{x}, t)=\boldsymbol{c}(\mathbf{x})+\sum_{j=1}^{q} \boldsymbol{\phi}_{j}(\mathbf{x}) a_{j}(t)
$$

Note that the POD is applied to an input collection that satisfies homogeneous Dirichlet boundary condition on $\Gamma_{D}$ and is divergence free. By linearity, these properties are transmitted to the POD basis vectors. Hence, taking $\boldsymbol{w}=\boldsymbol{\phi}_{i}$ for $i=1, \ldots, q$ in (2.6) and (2.7), one obtains

$$
\int_{\Omega}\left(\rho \frac{\partial \boldsymbol{u}^{r}}{\partial t}+\rho\left(\boldsymbol{u}^{r} \cdot \nabla\right) \boldsymbol{u}^{r}\right) \cdot \boldsymbol{\phi}_{i} \mathrm{~d} \Omega=-\int_{\Omega} \boldsymbol{\tau}\left(\boldsymbol{u}^{r}\right): \nabla \boldsymbol{\phi}_{i} \mathrm{~d} \Omega \quad \text { for } \quad i=1, \ldots, q .
$$

Note that the incompressibility constraint (2.7) is automatically satisfied since each $\boldsymbol{\phi}_{j}$ is solenoidal in the decomposition (2.17), and its associated Lagrange multiplier, the pressure, is eliminated from (2.6) or (2.9). Using the orthogonal decomposition (2.17) in the set of $q$ equations, (2.18) leads to a set of ODEs for the time coefficients $\boldsymbol{a}=\left[a_{1}, \ldots, a_{q}\right]^{T}$

$$
\dot{\boldsymbol{a}}(t)=f(\boldsymbol{a}(t)) \text {. }
$$

In the present study, (2.19) is integrated in time using the implicit second-order Crank-Nicolson scheme. Moreover, accurate initial conditions can be obtained from the DNS data, for an orthogonal basis: $a_{i}(0)=\left(\boldsymbol{y}(0, \cdot), \boldsymbol{\phi}_{i}(\cdot)\right)_{\Omega}$ for $i=1, \ldots, q$.

\subsubsection{Accuracy of ROM solutions}

We first consider the baseline flow at $R e=100$. After an initial settling, the flow reaches its periodic state in time. At this point, $m=58$ snapshots of the FE solution were collected over one vortex shedding cycle of period $T$ to build the snapshot data matrix. The fluctuating kinetic energy captured by these modes is illustrated by plotting the POD spectrum on a logarithmic scale in figure 3 . Observe that there is a rapid decrease in the energy distribution. Thus, the effectiveness of the POD in terms of energy representation as measured by (2.16) and given in table 2 is rapidly large. Indeed, the first six POD modes account for more than $99 \%$ of the flow energy. As a consequence, it was found by Deane et al. (1991) and Sirisup \& Karniadakis (2004), for the similar flows over a circular cylinder, that a six-mode POD system gives accurate results when compared with DNS data, at least for the short-term dynamics 


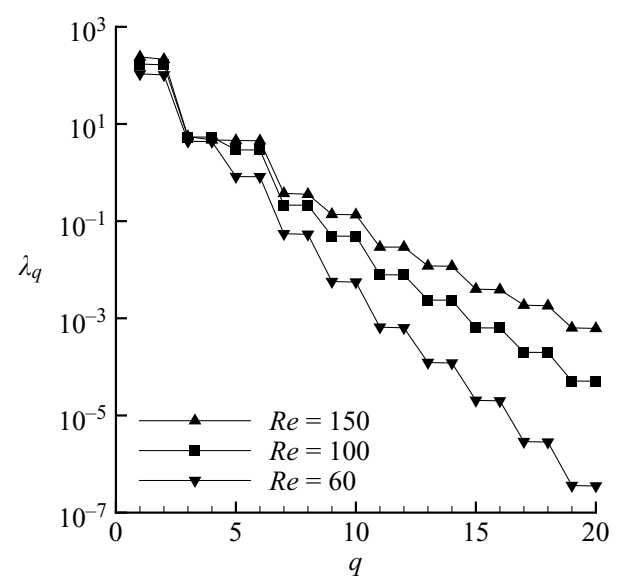

FIgURE 3. First 20 values of the POD spectrum for different Reynolds numbers.

as also reported by Galletti et al. (2004), Couplet et al. (2005) and Borggaard et al. (2007) for the case of a square cylinder at this Reynolds number. Figure 3 and table 2 also report results for $R e=150$ and $R e=60$. As can be seen, the lower the value of the Reynolds number, the steeper the slope of the spectrum and thus the better the efficiency of the POD. It means that a POD basis of fixed dimension $q$ accounts for a more important fraction of the energy in the data when $R e$ is low. This ordering is not true for the first three modes but then the picture is clear. Hence, it is expected that the POD-Galerkin models for a fixed dimension $q>3$ will be more accurate when $R e$ is low since the first $q$ POD modes contain more energy.

Figure 4 shows the contours of the streamwise component of the first six spatial POD vectors at the baseline. They are either symmetric or antisymmetric reflecting the topology of the vortex street and the geometric symmetry. However, it is not possible to foretell whether a mode will be even or odd in $y$ with the exception of the two primary modes. As noticed by Deane et al. (1991), their streamwise component is odd (see figure 4) and their normal component even because the fluctuating part of the flow snapshots appears clearly to possess this feature. However, the main characteristic in the decomposition is that the eigenvalues decay pairwise and that the POD modes can be grouped in pairs. These results are consistent with the topology of the vortex street (see e.g. Deane et al. 1991; Sirisup \& Karniadakis 2004; Couplet et al. 2005; Hay, Borggaard \& Pelletier 2008).

We now evaluate the accuracy of the approximated solution of the baseline flow obtained from the POD-based ROM during one flow period. The approximation to the full-order flow solution is expressed as a linear combination of the POD modes using (2.17). The time-dependent coefficients obtained from solving ROM (2.19) are denoted by $\left\{a_{i}^{r}\right\}_{i=1, q}$. The ROM solution $\boldsymbol{u}^{r}$ will be compared to the full-order solution obtained from the DNS $\boldsymbol{u}^{D N S}$. For the purpose of accessing the accuracy of the ROM, we also consider the approximated solution $\boldsymbol{u}^{p}$ as the best representation of $\boldsymbol{u}^{D N S}$ by the $q$-dimension POD basis using decomposition (2.17). Thus, for $\boldsymbol{u}^{p}$, the time coefficients, denoted by $\left\{a_{i}^{p}\right\}_{i=1, q}$, are obtained by projecting the DNS data on the reduced-POD basis. For an orthonormal basis, one has

$$
a_{i}^{p}(t)=\left(\boldsymbol{\phi}_{i}(\cdot), \boldsymbol{u}^{D N S}(t, \cdot)\right)_{\Omega} \quad \forall i=1, \ldots, q .
$$


(a)

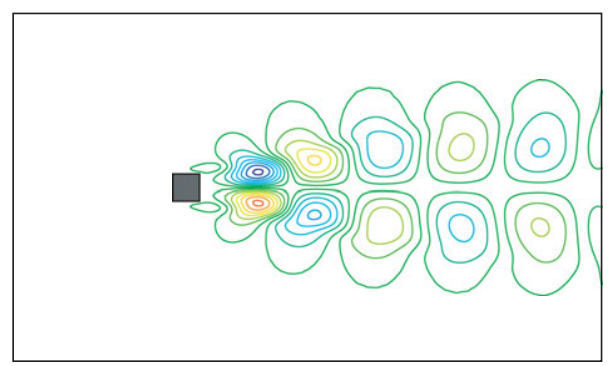

$\phi_{1}$

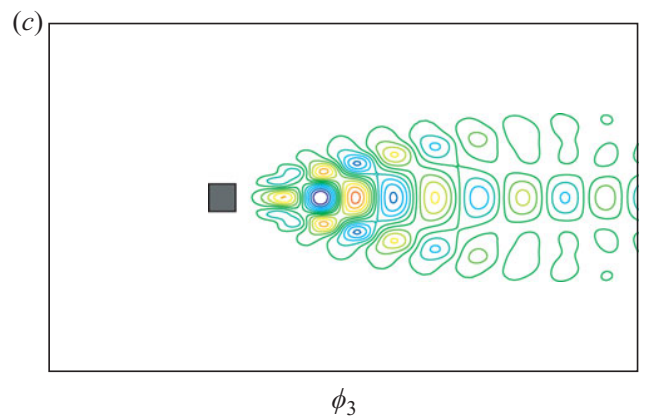

(e)

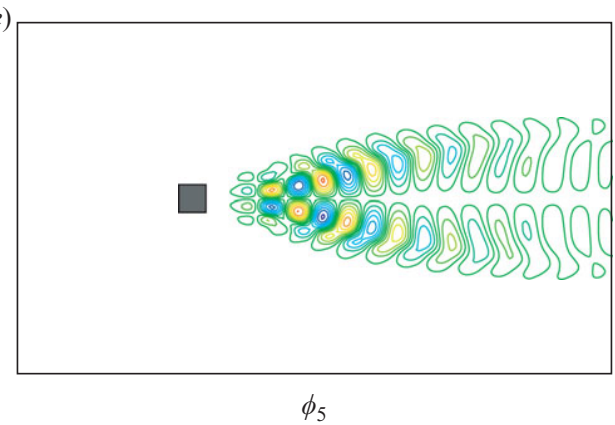

(b)

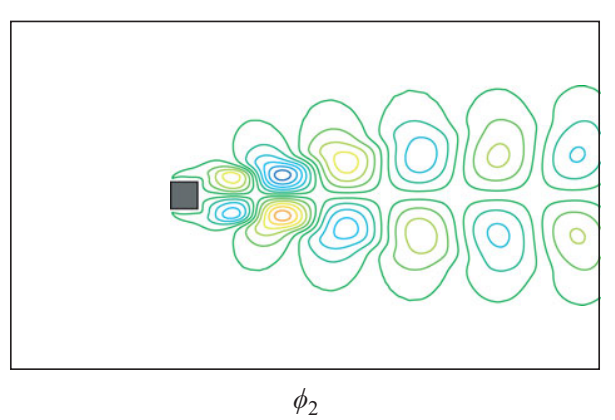

(d)

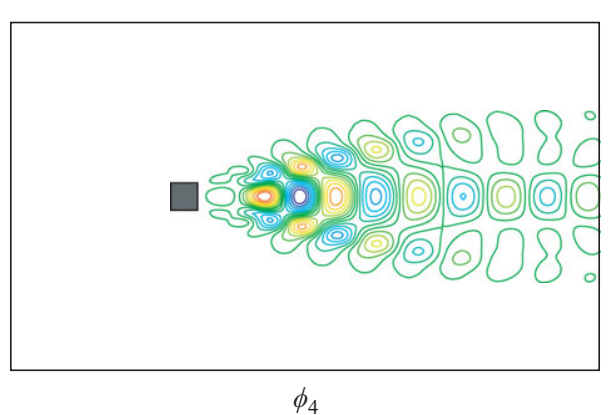

$(f)$

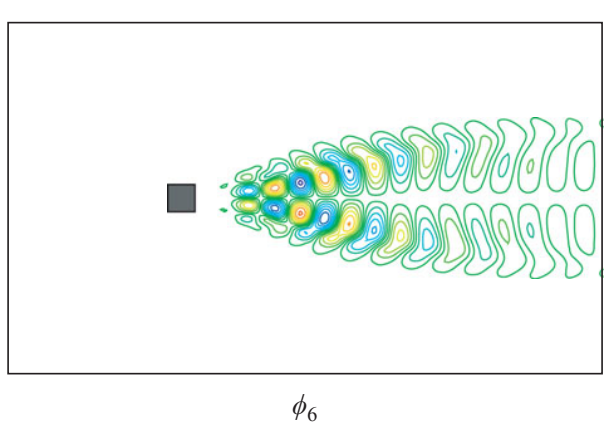

Figure 4. Iso-lines of the streamwise component of the first six POD modes.

Figure 5(a) shows the first four time coefficients using six POD modes in $\boldsymbol{u}^{r}$ and $\boldsymbol{u}^{p}$. As can been seen, the agreement between the $a_{i}^{r}$ and $a_{i}^{p}$ is very good. Note that the time coefficients can be grouped in pairs and each pair of coefficients are approximately sine and cosine functions of period $T / k$ with $k$ the pair wavenumber. We now compare the approximations $\boldsymbol{u}^{r}$ and $\boldsymbol{u}^{p}$ to $\boldsymbol{u}^{D N S}$ for different $q$-dimensional bases. For doing so, we compute the average relative errors in these approximations for each value of $q$ as

$$
\begin{aligned}
& e_{r e l}^{r}(\boldsymbol{u})=\left\langle\left\|\boldsymbol{u}^{r}-\boldsymbol{u}^{D N S}\right\|_{\Omega}\right\rangle /\left\langle\left\|\boldsymbol{u}^{D N S}\right\|_{\Omega}\right\rangle, \\
& e_{r e l}^{p}(\boldsymbol{u})=\left\langle\left\|\boldsymbol{u}^{p}-\boldsymbol{u}^{D N S}\right\|_{\Omega}\right\rangle /\left\langle\left\|\boldsymbol{u}^{D N S}\right\|_{\Omega}\right\rangle .
\end{aligned}
$$

Figure 5(b) shows the plot of $e_{r e l}^{r}$ and $e_{r e l}^{p}$ with the dimension $q$ of the considered basis. As can be seen, the relative errors in the approximated solutions are very close up to $q=26$. This result shows that the ROM performs well in computing the time 

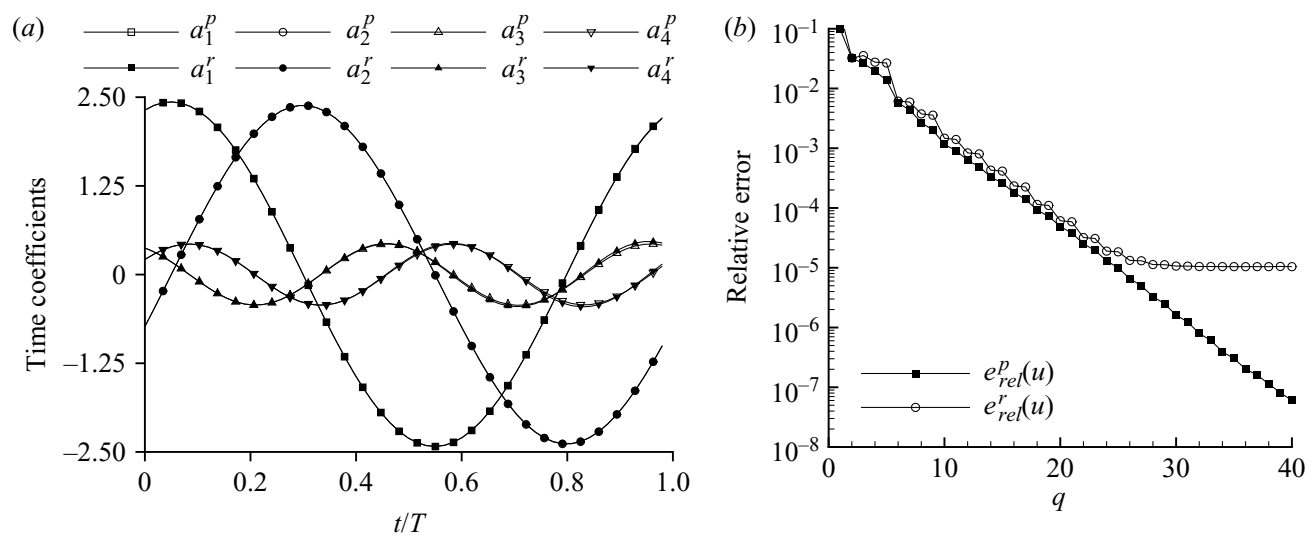

FIGURE 5. Performance of baseline reduced-order models at $R e=100$.

coefficients for decomposition (2.17). However, adding higher-order modes greater than $q=26$ in the ROM approximation does not improve the model solution. This occurs when the numerical error becomes larger than the modelling error in (2.17). This numerical error arises from the discrete sampling of the data over one period and from the spatial and temporal discretization. It is however considered sufficient since the approximated solution can reproduce the DNS data with four to five correct digits. It is worth noting that no calibration is used in the present study. Unlike the experiences reported in the literature (see e.g. Noack et al. 2003; Sirisup \& Karniadakis 2004; Bergmann et al. 2005), we have not observed any numerical instability after integration over a number of vortex shedding periods. During the first few periods, depending on the dimension of the basis used, the ROM solution slightly drifts away from the short-term dynamics shown in figure 5(a) for $q=6$ due to the truncation in the basis. However, once the low-dimensional limit cycle is reached, it never diverges from it even after integration over 25000 periods (we have tested models for $q=4$, 6,8 or 12). This is illustrated in figure 6 which shows the phase portrait of the first modes (the last 10 shedding periods are plotted). The higher the dimension, the better the approximation (and the less drifting from the short-term dynamics).

We now investigate the ability of the ROM built at $R e=100$ to predict solutions at different Reynolds numbers. To focus on the influence of the basis on the accuracy of the reduced-order solution, the initialization and centring are obtained from DNS data at the corresponding perturbed states. Figure 7 shows relative errors in reducedorder solutions with the Reynolds number for models of dimensions 6,12 and 24. At the baseline, the error is very low as already shown in figure $5(b)$. However, the error dramatically increases when the Reynolds number is changed. Furthermore, even for the smallest parameter perturbation $(R e \approx 95)$, the improvement of the approximated solution with the dimension of the basis stops. Hence, this increase in the error is not due to the truncation in the basis. These results simply show that as one goes further from the baseline in the parameter space, the baseline POD basis becomes less and less adequate to represent the perturbed flow solution and leads to poor reduced-order modelling.

The remainder of this article aims at improving the ROMs using sensitivity analysis. 

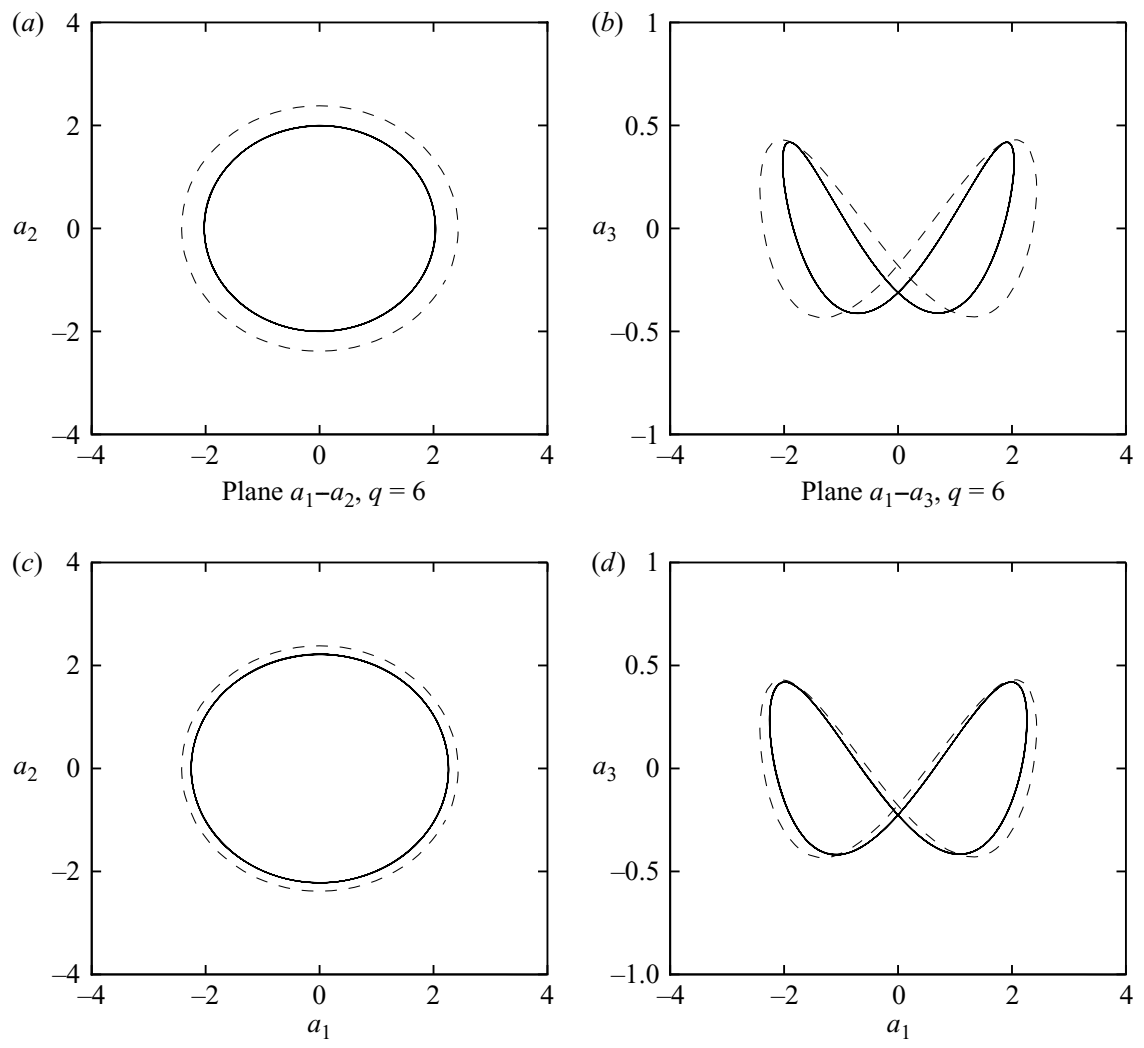

Plane $a_{1}-a_{2}, q=12$

Plane $a_{1}-a_{3}, q=12$

FIGURE 6. Limit cycle of dynamical systems at $R e=100$ (solid lines:

ROM, dashed lines: DNS).

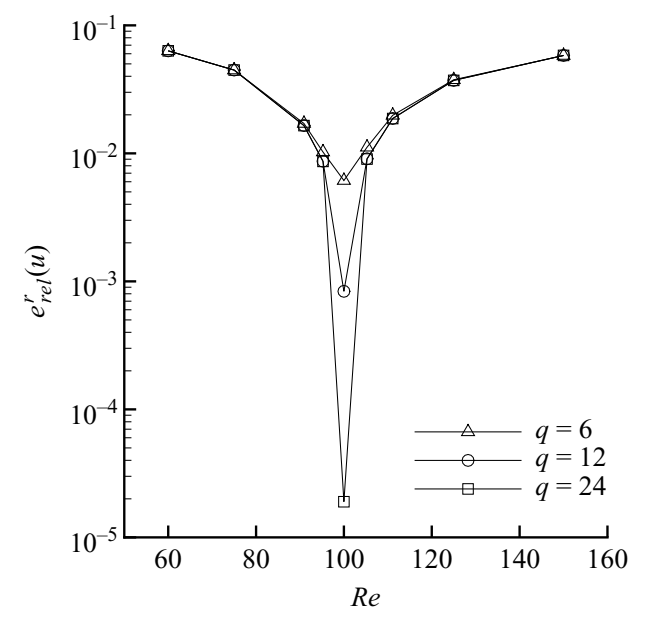

FIGURE 7. Relative errors in reduced-order modelled flows for different Reynolds numbers.

\section{Sensitivity analysis}

Sensitivity analysis has been an active topic of research in the past three decades since it finds many academic and industrial applications. A flow sensitivity is defined 
as the derivative of a flow variable with respect to a parameter in the system. It thus quantifies how the flow reacts to variations in the parameter. Among the main applications of sensitivity analysis are: fast evaluation of nearby configurations; uncertainty analysis of the system response; identification and ranking of key parameters and gradient computation for optimization algorithms. Furthermore, the body of work on sensitivity analysis has shown that it provides an enriched base of information on which to develop the understanding of complex flow problems.

\subsection{Flow sensitivity analysis}

The continuous sensitivity equations are derived formally by direct differentiation of the flow equations (2.1) and (2.2) with respect to an arbitrary parameter $\alpha$.

Thus, we treat the variable $\boldsymbol{u}$ as a function of both space and the parameter $\alpha$. This dependency is denoted by $\boldsymbol{u}=\boldsymbol{u}(\mathbf{x}, t ; \alpha)$. The flow sensitivities are defined as the partial derivatives

$$
\boldsymbol{s}_{\boldsymbol{u}}=\frac{\partial \boldsymbol{u}}{\partial \alpha} ; \quad \boldsymbol{s}_{p}=\frac{\partial p}{\partial \alpha} .
$$

Then, continuous equations governing sensitivity fields are written as

$$
\begin{aligned}
\rho^{\prime}\left[\frac{\partial \boldsymbol{u}}{\partial t}+(\boldsymbol{u} \cdot \nabla) \boldsymbol{u}\right]+\rho\left[\frac{\partial \boldsymbol{s}_{\boldsymbol{u}}}{\partial t}+\left(\boldsymbol{s}_{\boldsymbol{u}} \cdot \nabla\right) \boldsymbol{u}+(\boldsymbol{u} \cdot \nabla) \boldsymbol{s}_{\boldsymbol{u}}\right] & =\nabla \cdot \boldsymbol{\sigma}\left(\boldsymbol{s}_{\boldsymbol{u}}\right)+\nabla \cdot \boldsymbol{\tau}^{\prime}(\boldsymbol{u}), \\
\nabla \cdot \boldsymbol{s}_{\boldsymbol{u}} & =0
\end{aligned}
$$

where the derivatives of the fluid properties ( $\rho$ and $\mu$ here) are denoted by a (') and we define

$$
\begin{aligned}
\boldsymbol{\tau}^{\prime}(\boldsymbol{u}) & =\mu^{\prime}\left(\nabla \boldsymbol{u}+(\nabla \boldsymbol{u})^{T}\right) \\
\boldsymbol{\sigma}\left(\boldsymbol{s}_{\boldsymbol{u}}\right) & =-s_{p} \boldsymbol{I}+\mu\left(\nabla \boldsymbol{s}_{\boldsymbol{u}}+\left(\nabla \boldsymbol{s}_{\boldsymbol{u}}\right)^{T}\right) .
\end{aligned}
$$

To complete the description of the problem, the boundary conditions (2.4) and (2.5) have to be differentiated in the same way. Considering only value parameters (i.e. parameters that do not influence the geometry), one has

$$
\begin{aligned}
\boldsymbol{s}_{\boldsymbol{u}}=\boldsymbol{s}_{\boldsymbol{u}} & \left(\Gamma_{D}\right), \\
\left(\boldsymbol{\sigma}\left(\boldsymbol{s}_{\boldsymbol{u}}\right)+\boldsymbol{\tau}^{\prime}(\boldsymbol{u})\right) \cdot \boldsymbol{n}=\mathbf{0} & \left(\Gamma_{N}\right) .
\end{aligned}
$$

The weak forms of (3.2) and (3.3) are formed and solved with the same numerical methods as for the flow. Details on the methodology and numerical procedure can be found in the literature (see Hristova et al. 2006; Pelletier et al. 2008).

In continuation of the previous study, we consider the dynamic viscosity as a parameter to control the Reynolds number and compute the flow sensitivity with respect to $\mu$. Hence, we set $\mu^{\prime}=1$ and $\rho^{\prime}=0$. Furthermore, for this parameter, the Dirichlet boundary condition on $\Gamma_{D}$ for the sensitivity problem is homogeneous since $s_{u}=\mathbf{0}$.

Using a classical verification procedure in sensitivity analysis, we compare the solution of the sensitivity equation method presented above with the sensitivity computed by finite-difference (FD) of two nearby flow solutions

$$
\left.\frac{\partial \boldsymbol{u}}{\partial \mu}\right|_{F D}=\frac{\boldsymbol{u}\left(\mu_{0}+\Delta \mu\right)-\boldsymbol{u}\left(\mu_{0}\right)}{\Delta \mu}
$$

at the nominal parameter $\mu_{0}$. The parameter increment $\Delta \mu$ is chosen sufficiently small for the FD computation to be accurate and sufficiently large for the difference between the two nearby flow solutions to be at least one order of magnitude larger 

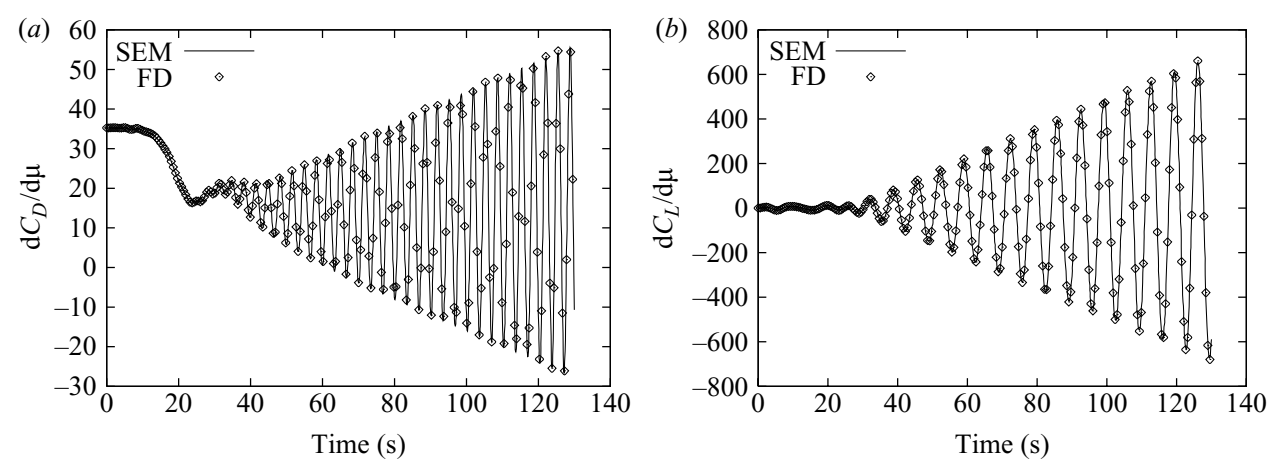

FIGURE 8. Comparison between FD and SEM.

than the discretization error. Here, we have found $\Delta \mu / \mu_{0}=0.01 \%$. We look at the sensitivity of the drag and lift coefficients that are related to the sensitivity of the loads applied by the flow to the cylinder as defined by

$$
\begin{aligned}
\frac{\partial}{\partial \mu}\left[\begin{array}{l}
C_{D} \\
C_{L}
\end{array}\right] & =\frac{\partial}{\partial \mu}\left\{\frac{1}{(1 / 2) \rho U_{\infty}^{2} D} \int_{\Gamma_{w}} \boldsymbol{\sigma}(\boldsymbol{u}) \cdot \boldsymbol{n} \mathrm{d} \Gamma\right\} \\
& =\frac{1}{(1 / 2) \rho U_{\infty}^{2} D} \int_{\Gamma_{w}}\left(\sigma\left(\boldsymbol{s}_{\boldsymbol{u}}\right)+\boldsymbol{\tau}^{\prime}(\boldsymbol{u})\right) \cdot \boldsymbol{n} \mathrm{d} \Gamma
\end{aligned}
$$

where $\Gamma_{w}$ is the square cylinder wall. All these results are shown in figure 8 . As can be seen, the agreement between the SEM of the FD solution is very good which shows that an accurate flow sensitivity is obtained for the problem at hand.

It is worth noting that the SEM provides an efficient way to generate flow sensitivities compared to the FD approach which is more straightforward since it only requires evaluations from the flow solver and thus almost no additional development. However, this option is costly because the problem must be solved for two or more values (for higher order finite differences when necessary) of each parameter. Furthermore, numerical evaluations of certain sensitivities may be limited for shape parameters since technical problems arise due to mesh topology changes. Finally, they may suffer from round-off errors (i.e. subtractive cancellation errors). The SEM has none of these limitations. Furthermore, the sensitivity problem is always linear and thus can be solved for a fraction of the cost of solving the nonlinear Navier-Stokes equations (see Ilinca et al. 2008; Pelletier et al. 2008 for a more complete discussion).

\subsection{POD sensitivity analysis}

This section aims at deriving the sensitivity of the discrete POD modes with respect to a generic value parameter $\alpha$. Sensitivity analysis of eigenvalue problems has been the subject of several studies in the literature. The methodology for computing the sensitivity of eigenvalues and eigenvectors of multiplicity one is reported by Fox \& Kapoor (1968) and Murthy \& Haftka (1988). The treatment of general multiple eigenvalues has been examined by Lancaster (1964) and, in the context of structural vibration problems, by Seyranian, Lund \& Olhoff (1994).

For the present discussion, we consider matrices with simple eigenvalues. This may seem to contradict the fact that the eigenvalues of the exact spatial autocorrelation tensor $R^{s}$, built from the analytical solution of the unsteady Navier-Stokes equations, are of multiplicity two. This is due to the spatio-temporal symmetry 
$[u(x, y, t), v(x, y, t)]^{T}=[u(x,-y, t+T / 2),-v(x,-y, t+T / 2)]^{T}$ (Deane et al. 1991). It is worth mentioning that the multiplicity of the eigenvalues is preserved through a change in the viscosity coefficient since, for the range of Reynolds numbers considered in this study, the periodic vortex shedding is always observed and hence the nature of the data is preserved. However, in the finite precision setting, this is only approximately true. Indeed, it suffices to use an unsymmetrical mesh in the DNS for this property to be only approximately observed. Hence, the discrete numerical approximation of $R^{s}$ has only single eigenvalues though they can clearly be grouped in pairs as already mentioned in $\S 2.3 .3$. Note that the treatment of multiple eigenvalues is not much more involved as long as the multiplicity is preserved with parameter changes. The interested reader is referred to Hay et al. (2008) for this case.

We start with (2.15) noting $\boldsymbol{B}=\boldsymbol{A}^{T} \boldsymbol{A}$; then the $k$ th column of $\boldsymbol{V}$, denoted by $\boldsymbol{V}_{k}$, is the solution of the eigenvalue problem

$$
\boldsymbol{B} \boldsymbol{V}_{k}=\lambda_{k} \boldsymbol{V}_{k} \text {. }
$$

We assume that the entries of the $\boldsymbol{B}, \boldsymbol{V}$ and $\boldsymbol{\Lambda}$ matrices are smooth functions of the parameter $\alpha$ so that (3.8) can be differentiated with respect to $\alpha$. For the sake of compactness, the partial derivative of any matrix or vector is denoted using the superscript $(\alpha)$. Thus, implicit differentiation of (3.8) with respect to $\alpha$ leads to

$$
\begin{aligned}
\boldsymbol{B}^{\alpha} \boldsymbol{V}_{k}+\boldsymbol{B} \boldsymbol{V}_{k}^{\alpha} & =\lambda_{k}^{\alpha} \boldsymbol{V}_{k}+\lambda_{k} \boldsymbol{V}_{k}^{\alpha}, \\
\left(\boldsymbol{B}-\lambda_{k} \boldsymbol{I}\right) \boldsymbol{V}_{k}^{\alpha} & =-\left(\boldsymbol{B}^{\alpha}-\lambda_{k}^{\alpha} \boldsymbol{I}\right) \boldsymbol{V}_{k} .
\end{aligned}
$$

Hence, the sensitivity of the vector $\boldsymbol{V}_{k}$ is solution to the symmetric linear system (3.10) which has solutions only if the right-hand side vector belongs to the range of $\boldsymbol{B}-\lambda_{k} \boldsymbol{I}$ and thus must be orthogonal to $\operatorname{ker}\left(\boldsymbol{B}-\lambda_{k} \boldsymbol{l}\right)$ which is generated by $\boldsymbol{V}_{k}$. In other words

$$
\boldsymbol{V}_{k}^{T}\left(\boldsymbol{B}^{\alpha}-\lambda_{k}^{\alpha} \boldsymbol{I}\right) \boldsymbol{V}_{k}=0 .
$$

Hence, since $\boldsymbol{V}_{k}$ has unit norm, the sensitivity of the eigenvalues is obtained through (Lancaster 1964; Fox \& Kapoor 1968; Murthy \& Haftka 1988; Seyranian et al. 1994)

$$
\lambda_{k}^{\alpha}=\boldsymbol{V}_{k}^{T} \boldsymbol{B}^{\alpha} \boldsymbol{V}_{k}
$$

Thus, solutions to system (3.10) are completely characterized. We found one particular solution, noted $s_{k}$ by solving it in the least-square sense (obtaining the minimum norm solution). Since $\lambda_{k}$ is simple, $s_{k}+\gamma \boldsymbol{V}_{k}$ for all $\gamma \in \mathbb{R}$ is the general expression for the solutions of (3.10). To determine the particular solution of (3.10) that corresponds to the sensitivity of $\boldsymbol{V}_{k}$, an additional condition must apply for finding the $\gamma$ that gives $\boldsymbol{V}_{k}$. It comes naturally from the normalization condition $\boldsymbol{V}_{k}^{T} \boldsymbol{V}_{k}=1$ which was used to specify $\boldsymbol{V}_{k}$. Differentiating the normalization condition gives $\boldsymbol{V}_{k}^{T} \boldsymbol{V}_{k}^{\alpha}=0$ and thus we find

$$
\boldsymbol{V}_{k}^{\alpha}=s_{k}-\left(s_{k}^{T} \boldsymbol{V}_{k}\right) \boldsymbol{V}_{k} \quad \text { and } \quad \gamma=-s_{k}^{T} \boldsymbol{V}_{k}
$$

Once the sensitivity of the matrices $\boldsymbol{V}$ and $\boldsymbol{\Lambda}$ are determined, the sensitivity of the POD spatial modes are easily computed by differentiating $\boldsymbol{\Phi}=\boldsymbol{Y} \boldsymbol{V} \boldsymbol{\Lambda}^{-1 / 2}$

$$
\begin{aligned}
\boldsymbol{\Phi}^{\alpha} & =\boldsymbol{Y}^{\alpha} \boldsymbol{V} \boldsymbol{\Lambda}^{-1 / 2}+\boldsymbol{Y} \boldsymbol{V}^{\alpha} \boldsymbol{\Lambda}^{-1 / 2}+\boldsymbol{Y} \boldsymbol{V}\left(\boldsymbol{\Lambda}^{-1 / 2}\right)^{\alpha} \\
& =\boldsymbol{Y}^{\alpha} \boldsymbol{V} \boldsymbol{\Lambda}^{-1 / 2}+\boldsymbol{Y} \boldsymbol{V}^{\alpha} \boldsymbol{\Lambda}^{-1 / 2}-\frac{1}{2} \boldsymbol{Y} \boldsymbol{V}\left(\boldsymbol{\Lambda}^{-1 / 2} \boldsymbol{\Lambda}^{\alpha} \boldsymbol{\Lambda}^{-1}\right) \\
& =\boldsymbol{Y}^{\alpha} \boldsymbol{V} \boldsymbol{\Lambda}^{-1 / 2}+\boldsymbol{Y} \boldsymbol{V}^{\alpha} \boldsymbol{\Lambda}^{-1 / 2}-\frac{1}{2} \boldsymbol{\Phi} \boldsymbol{\Lambda}^{\alpha} \boldsymbol{\Lambda}^{-1} .
\end{aligned}
$$




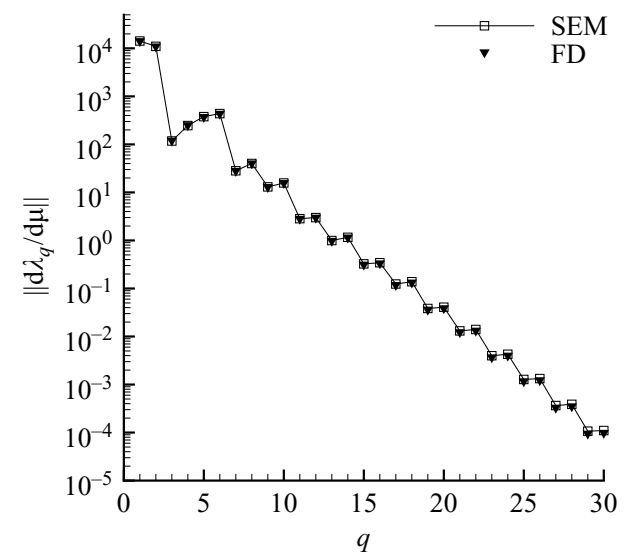

Figure 9. Absolute value of the first 30 values of the sensitivity POD spectrum.

We note that this discussion could have been carried out from the infinitedimensional setting with minor changes related to the fact that the eigenspaces are then two-dimensional. There are interesting differences between the continuous and discrete settings when the parameter influences the shape of the domain. This will be studied in future work.

It is worth noting that in (3.13) the mode sensitivities $\boldsymbol{\Phi}^{\alpha}$ are evaluated from a linear combination of the flow data, the sensitivity data and the original POD modes. Hence, the sensitivities of the modes share their common linear properties. As the centred flow data and the POD modes, the sensitivity data have zero values on the boundary $\Gamma_{D}$ and are solenoidal (see $\S 3.1$ ). Thus, the mode sensitivities also do, implying that (2.18) is still valid if the mode sensitivities are used as test functions in the Galerkin method.

Before ending this section, we emphasize that the mode sensitivities may not always be defined. Indeed, we have here assumed that data snapshots, eigenfunctions and eigenvalues are smooth functions of the parameter in the neighbourhood of the current state so that (3.8) can be differentiated. Clearly, there is a number of situations for which this is not true. This is a limitation of this method. For the case of the flow past a cylinder, the POD modes do not undergo a smooth transition at the first Hopf bifurcation (see $\S 2.2$ ) so that the mode sensitivities are not defined at this Reynolds number.

\subsection{Numerical results}

The sensitivity of the snapshot data matrix $\boldsymbol{Y}^{\alpha}$ is built by assembling $m=58$ snapshots of the FE sensitivity solution collected at the same instants as the flow snapshots. Then, $\boldsymbol{A}^{\alpha}=\boldsymbol{L}^{T} \boldsymbol{Y}^{\alpha}$ and $\boldsymbol{B}^{\alpha}=\boldsymbol{A}^{\alpha T} \boldsymbol{A}+\boldsymbol{A}^{T} \boldsymbol{A}^{\alpha}$ are formed and the sensitivity of the eigenvalues and POD modes is computed. We recall that the mass matrix $\boldsymbol{M}$ (and thus $\boldsymbol{L}$ ) does not depend on the parameter $\alpha=\mu$ under consideration here since it is a value parameter. Figure 9 shows the absolute value of the sensitivity of the POD spectrum. For verification purposes, we compare the results obtained by the method described in $\S 3.2$ (we refer to them as SEM) to the sensitivity computed by finite-difference (FD) using the decompositions of the baseline $\left(\mu_{0}\right)$ and perturbed flows $\left(\mu_{0}+\Delta \mu\right)$ with again $\Delta \mu / \mu_{0}=0.01 \%$

$$
\left.\frac{\partial \lambda_{k}}{\partial \mu}\left(\mu_{0}\right)\right|_{F D}=\frac{\lambda_{k}\left(\mu_{0}+\Delta \mu\right)-\lambda_{k}\left(\mu_{0}\right)}{\Delta \mu} .
$$




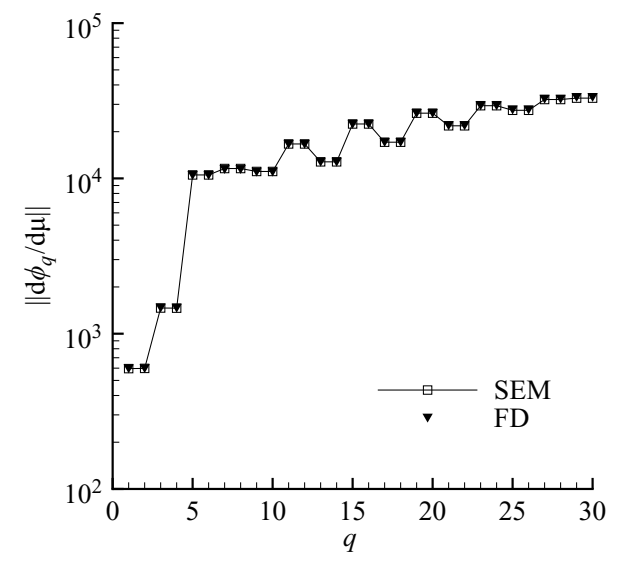

FIGURE 10. Norm of the first 30 POD mode sensitivities.

As can be seen, the agreement between the two approaches is very good. Indeed, for the first 14 eigenvalue sensitivities, the relative differences are less than $2 \%$ and reach $5 \%$ for the first 20. Except for the fifth and sixth modes, the eigenvalue sensitivity (roughly) decreases with the mode number in a similar way as the eigenvalues do. This shows that the ranking of the eigenvalues will be preserved through changes in the Reynolds number. Only the rate of the energy decay will be affected so that the dimension of the basis required to represent a certain amount of the total energy will change as already noted in $\S 2.3 .3$. However, the magnitudes of the sensitivities of the fifth and sixth eigenvalues are larger than the magnitudes of the sensitivities of the third and fourth eigenvalues. Given that the eigenvalues between 3 and 6 are very close (see figure 3), it indicates that the fifth and sixth POD spatial functions play more significant roles than the third and fourth POD spatial functions in capturing energy at nearby parameter values. This information could be used to guide the basis truncation criteria. Indeed, in our case, the sensitivity information provides the insight that we would not want to truncate between 3 and 6 . Note that the sensitivities can also be grouped in pairs which shows that this property of the eigenvalues is preserved through a change in the viscosity around the baseline as expected from the previous discussion. It is worth noting that all the eigenvalue sensitivities but the fourth are negative. This result was also expected since a positive perturbation of the parameter increases the viscosity so that the system is likely to dissipate more energy and thus the positively perturbed flows have less energy than the baseline flow.

To assess the accuracy of the POD mode sensitivities, figure 10 compares the norm of the first 30 POD mode sensitivities computed with their FD counterparts. Observe that the agreement is very good. The first 18 sensitivities exhibit less than $2 \%$ of the relative differences and the first 36 exhibit less than $5 \%$. A qualitative picture of the structure of the POD mode sensitivities is given in figure 11 where contours of the streamwise component of the first six POD mode sensitivities is presented. Clearly, they greatly resemble the first six POD modes presented in figure 4 up to a switch between the modes within each pair. This apparent similarity is due the property of homogeneity in the time direction which makes the temporal eigenfunctions $\boldsymbol{\psi}_{i}$ Fourier modes (once again this is only approximately true in finite precision). Since they are approximately a sine or cosine function of period $T / k$ with $k$ the pair wavenumber, their derivatives with respect to any parameter are approximately a linear combination of these sine and cosine functions. Each sensitivity is made 
(a)

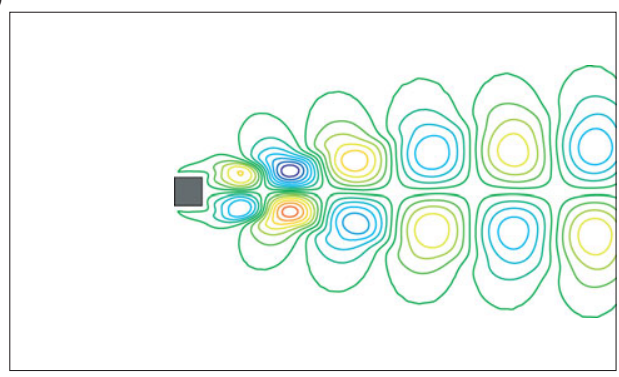

$\partial \phi_{1} / \partial \mu$

(c)

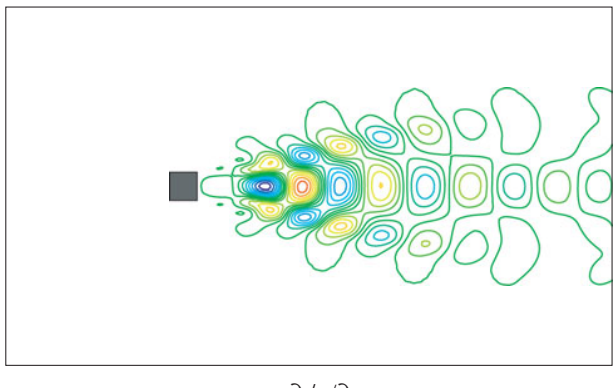

$\partial \phi_{3} / \partial \mu$

(e)

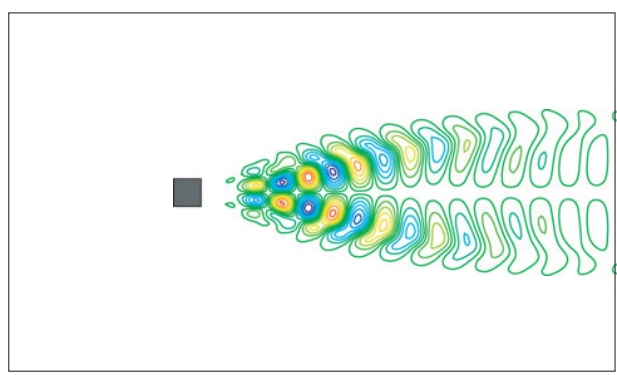

$\partial \phi_{5} / \partial \mu$ (b)

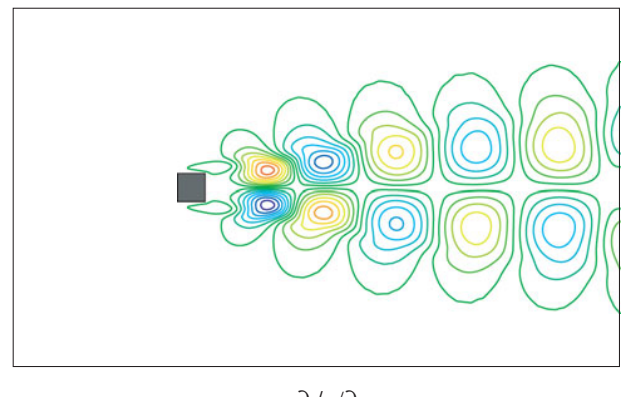

(d)

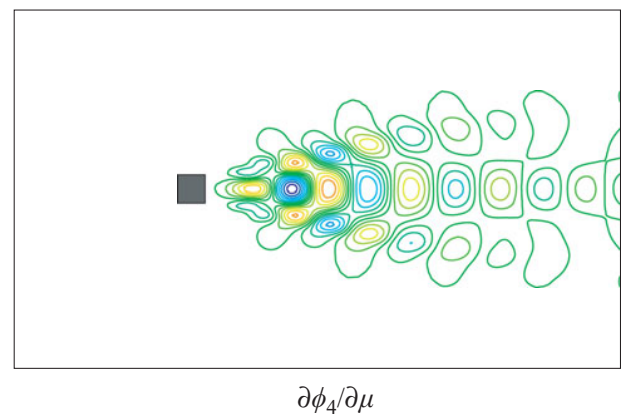

$(f)$

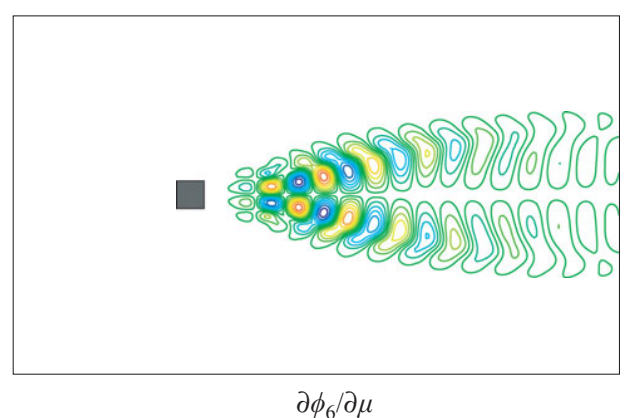

FIGURE 11. Iso-lines of the sensitivity of the streamwise component of the first six POD modes.

orthogonal to its corresponding original function through (3.12). Thus, if $\boldsymbol{\psi}_{i}$ is a sine function, its derivative is a cosine function and vice versa (for more details see Hay et al. 2008).

However, the similarity of the POD modes and their sensitivities is only a visual artefact since they can be shown to be linearly independent and thus they span different subspaces. To see this, we consider the set of vectors containing the POD modes and their sensitivities and we make them maximally linearly independent by computing an orthonormal basis for this set of vectors. The eigenfunctions are not modified from this operation since they already form a set of linearly independent orthonormal vectors. Components of the POD modes are removed from the sensitivities. Contours of the streamwise component of the resulting orthogonalized 
(a)

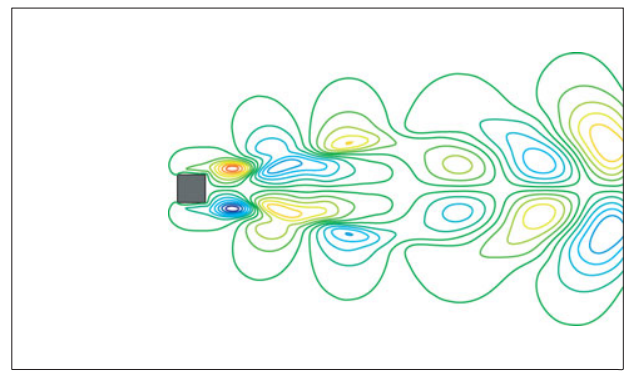

$\partial \phi_{1} / \partial \mu$

(c)

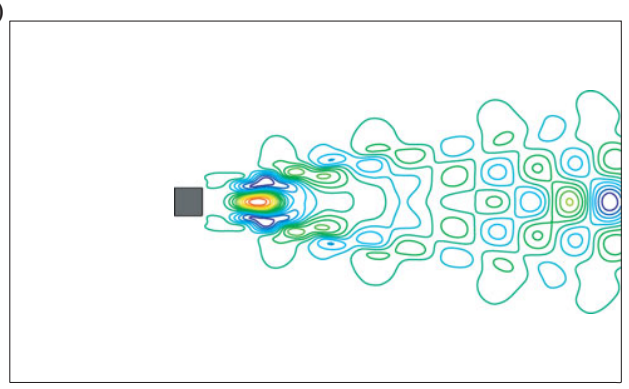

$\partial \phi_{3} / \partial \mu$

(e)

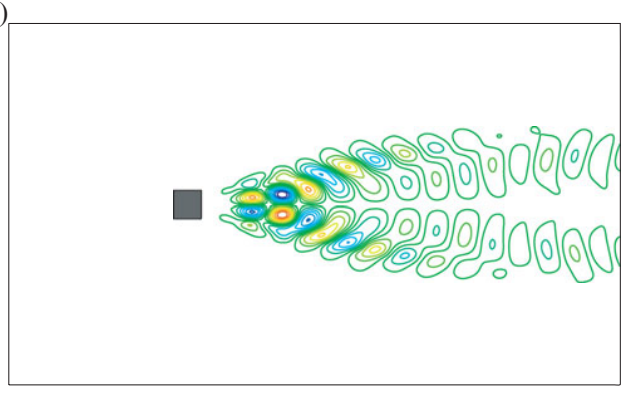

$\partial \phi_{5} / \partial \mu$ (b)

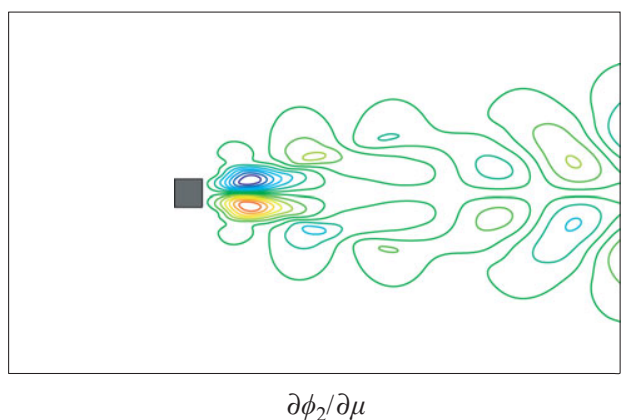

(d)

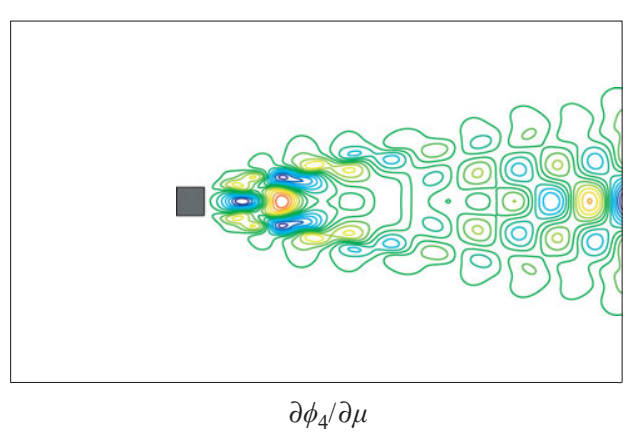

$(f)$

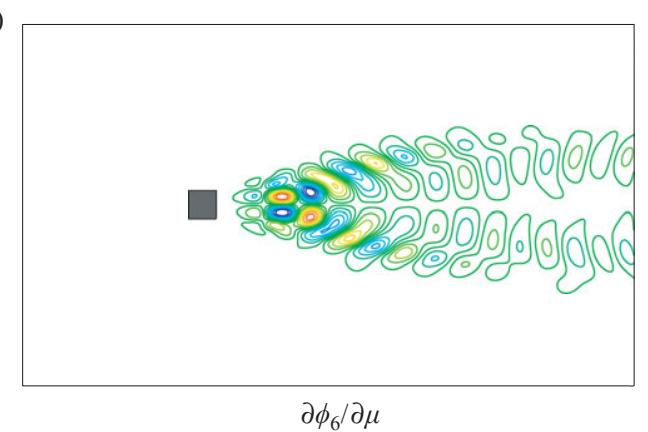

FIGURE 12. Iso-lines of the orthogonalized sensitivity of the streamwise component of the first six POD modes.

sensitivities are shown in figure 12 for the first six modes. Their structures are now clearly different of those of the original modes. However, they also exhibit antisymmetry or symmetry with respect to the mid-line of the geometry. This was expected since the nature of the flow is preserved for a wide range of Reynolds number around the baseline.

\section{More robust reduced-order models}

This section aims to improve the robustness of the bases used to construct approximated flow solutions by using the now available POD mode sensitivities.

\subsection{More robust reduced bases}

We examine two different ideas for constructing improved reduced bases 
(a) Extrapolated basis: as for the flow variables, we treat each POD mode as a function of both space and parameter $\alpha: \boldsymbol{\phi}=\boldsymbol{\phi}(\mathbf{x} ; \alpha)$. In the context of the cylinder flow, this is a natural idea since the POD modes clearly depend on the Reynolds number. A change $\Delta \alpha$ in the parameter from its baseline value $\alpha_{0}$ is reflected in the modes through a first-order expansion in the parameter space

$$
\boldsymbol{\phi}(\mathbf{x} ; \alpha)=\boldsymbol{\phi}\left(\mathbf{x} ; \alpha_{0}\right)+\Delta \alpha \frac{\partial \boldsymbol{\phi}}{\partial \alpha}\left(\mathbf{x} ; \alpha_{0}\right)+O\left(\Delta \alpha^{2}\right) .
$$

The capability of this extrapolation clearly depends on whether or not the POD modes exhibit a nearly linear dependence with respect to the parameter $\alpha$. However, the dimension of the reduced basis is preserved and the reduced approximation of the flow variables is still expressed using (2.17). Other approaches based on the idea of mode interpolation can be found in the literature (Lehmann et al. 2005; Lieu et al. 2006; Morzyński et al. 2007). It has been shown that they lead to an increased robustness of the derived POD models with parameter changes and thus motivate the extrapolation approach using mode sensitivities.

(b) Expanded basis: the sensitivities of the modes have been shown to span a different subspace than the POD modes. Thus, it is natural to expect that if the approximated solution is selected in the union of the two subspaces a broader class of solution can be represented. We expand the original basis to consist of the first $q$ eigenfunctions with their $q$ sensitivities $\left[\boldsymbol{\phi}_{1} ; \ldots ; \boldsymbol{\phi}_{q} ; \boldsymbol{\phi}_{1}^{\alpha} ; \ldots ; \boldsymbol{\phi}_{q}^{\alpha}\right]$. The underlying assumption behind this approach is that the subspace spanned by the mode sensitivities is well suited to address the change in the solutions induced by a change in the parameter. This is indeed a legitimate assumption since the sensitivities, being the first-order derivatives, represent the preferred directions in phase space where parameter changes occur. However, the dimension of the reduced basis has now doubled and the reduced approximation of the flow variables is now expressed as

$$
\boldsymbol{u}^{r}(\mathbf{x}, t)=\boldsymbol{c}(\mathbf{x})+\sum_{j=1}^{q} \boldsymbol{\phi}_{j}(\mathbf{x}) a_{j}(t)+\sum_{j=q+1}^{2 q} \boldsymbol{\phi}_{j-q}^{\alpha}(\mathbf{x}) a_{j}(t) .
$$

We first look at the ability of these two new sets of functions to span the data obtained at Reynolds numbers other than $R e_{0}$. To do so, we project the flow snapshots obtained at various Reynolds numbers onto the $q$-dimensional baseline and extrapolated POD bases and the $2 q$-dimensional expanded basis to produce the time coefficients in (2.17) and (4.2). Then we measure the discrepancies between the resulting approximated flows and DNS data from (2.22) in figure 13. Note that in order to isolate the influence of the reduced-basis, the centring trajectory $\boldsymbol{c}(\boldsymbol{x})$ in $(2.17)$ and (4.2) is defined as the time average of flow snapshots at each perturbed state. As can be seen in figure 13, the two new bases are better-suited than the baseline basis to represent the data at the perturbed states. For the extrapolated approach, the improvement is only significant locally around the baseline which is consistent with the first-order extrapolation idea. The improvement is also better for the 6dimensional model than that for the 12-dimensional model. This is due to the fact that the lower the mode the better its linear extrapolation in the parameter space. That is the first POD modes exhibit a more linear dependency with the viscosity than the later higher order modes. However, the best modelling capability is obtained from the expanded POD basis. Compared to the extrapolated basis, the expanded basis performs significantly better because the influence of the mode sensitivities in the linear combination (4.2) is not constrained to be a fraction of the weight 

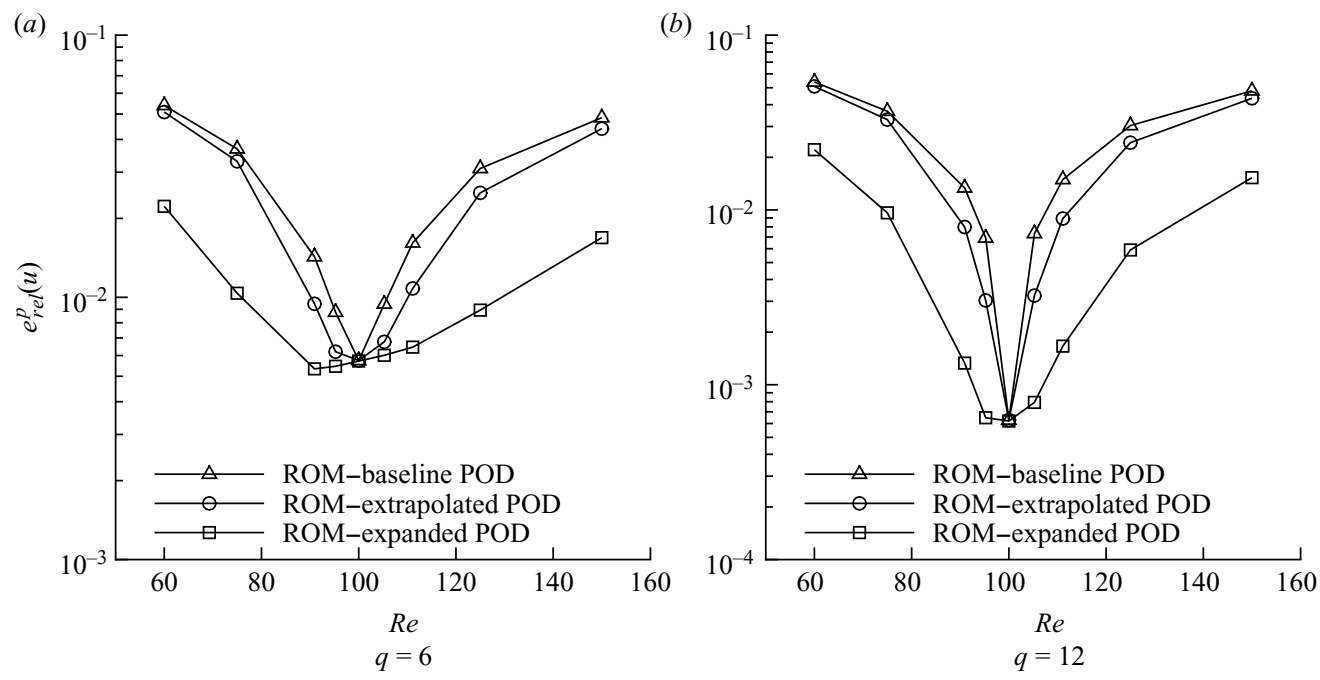

FIGURE 13. Relative errors in approximated (projected) flows using different bases.

of its corresponding POD mode following linear extrapolation. Again the closer to the baseline, the larger the improvement. However, as opposed to the extrapolated approach, the expanded basis approximation yields a significant improvement even for the largest parameter perturbations regardless of the basis dimension.

\subsection{Accuracy of the sensitivity-based low-dimensional modelling}

This section reports the accuracy of the ROMs built from the improved reduced bases described previously for the short-term dynamics of the flow. As previously, to focus on the influence of the basis used in the reduced-order modelling, all ROM simulations are initialized using the DNS data at the considered state and use the appropriate centring.

Figure 14 shows the relative errors in reduced-order modelled flows using different bases as calculated from (2.21). Generally speaking, the relative errors are close to what was reported for the projection test in figure 13. This means that the dynamical systems obtained by projection of the Navier-Stokes equations on the bases can efficiently reproduce the short-term dynamics of all the components in the bases (baseline modes, extrapolated modes, baseline mode sensitivities) for all Reynolds numbers. This is not an obvious result especially considering that the sensitivity of the baseline modes has never been studied in the literature. Indeed, there is no guarantee that a Galerkin-based dynamical system can reproduce the dynamics of its modal components even with correct initial conditions. The only important difference with the projection results above appears for the expanded approach with $q=6$ and close to the baseline where it performs slightly worse. Particularly, at the baseline $\left(\operatorname{Re}_{0}=100\right)$, the $(2 q=12)$-dimensional expanded basis, which contains the first six baseline POD modes, brings a solution relative error slightly higher than when only the first six modes are included (baseline approach). This means that an amplitude-selection mechanism in this dynamical system has incorrectly transferred energy to some mode sensitivities though they represent an insignificant fraction of the flow energy at the baseline. However, this phenomenon is indeed limited and is not observed for $q=12$. All in all, the expanded approach performs significantly better than the baseline approach. 

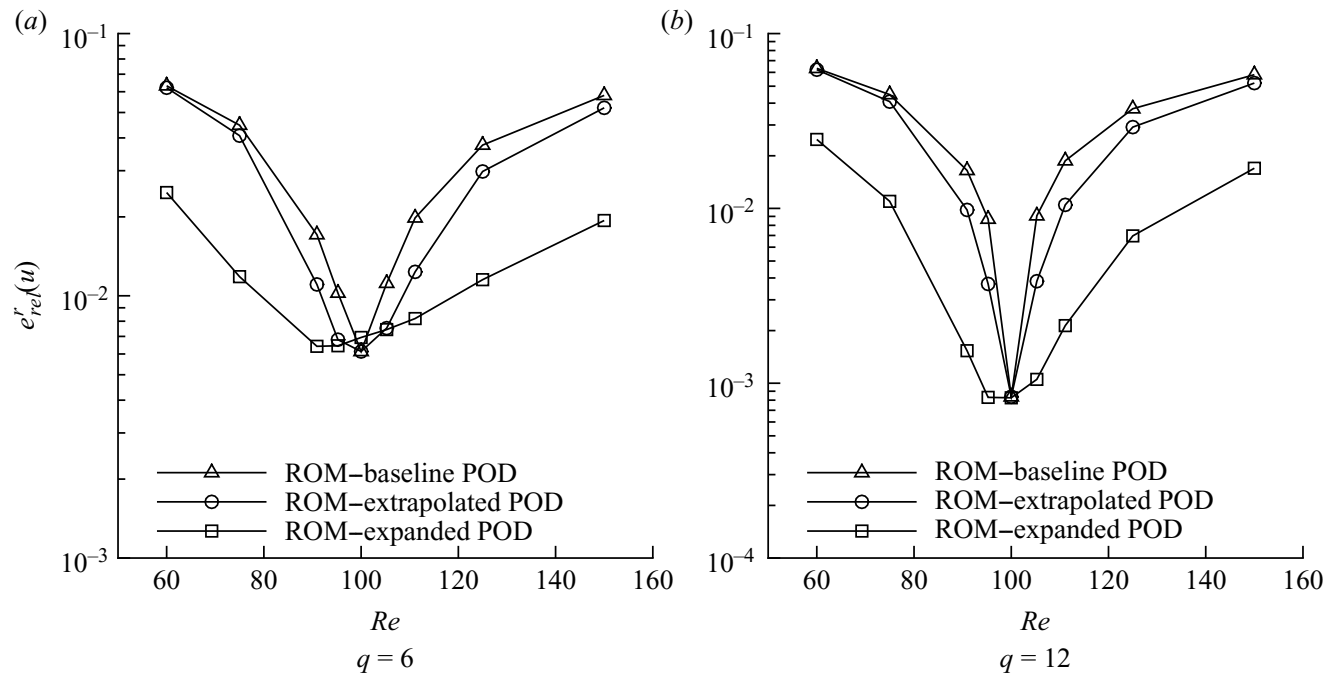

FIGURE 14. Relative errors in reduced-order modelled flows using different bases.

\subsection{Low-dimensional modelling and long-term dynamics}

In this section, we build low-dimensional model using only DNS data extracted at the baseline Reynolds number. That is, the initial conditions, centring trajectories and bases are only calculated from the DNS data at the baseline parameter value. Furthermore, we look at the long-term behaviour of the low-dimensional systems by integrating them over a sufficiently long time to reach their attractors. This is a more involved problem than above since transients of dynamical systems initialized from the projection on the attractor of the full-order simulation can stay close to it over several periods but can drift away after a sufficient long time (Deane et al. 1991).

Using the ideas behind the extrapolated approach, the centring is linearly extrapolated in the parameter space to the perturbed state $\left(\mu_{0}+\Delta \mu\right)$. In the expanded approach, the sensitivity of the centring mode is added to the low-dimensional basis. Hence, the modelled solutions are expressed as

(a) Baseline approach (dimension $q$ )

$$
\boldsymbol{u}^{r}=\boldsymbol{c}_{\mu_{0}}+\sum_{j=1}^{q}\left[\boldsymbol{\phi}_{j}\right]_{\mu_{0}} a_{j}
$$

(b) Extrapolated approach (dimension $q$ )

$$
\boldsymbol{u}^{r}=\left(\boldsymbol{c}_{\mu_{0}}+\Delta \mu \boldsymbol{c}_{\mu_{0}}^{\alpha}\right)+\sum_{j=1}^{q}\left(\left[\boldsymbol{\phi}_{j}\right]_{\mu_{0}}+\Delta \mu\left[\boldsymbol{\phi}_{j}^{\alpha}\right]_{\mu_{0}}\right) a_{j},
$$

(c) Expanded approach (dimension $2 q+1$ )

$$
\boldsymbol{u}^{r}=\boldsymbol{c}_{\mu_{0}}+\sum_{j=1}^{q}\left[\boldsymbol{\phi}_{j}\right]_{\mu_{0}} a_{j}+\sum_{j=q+1}^{2 q}\left[\boldsymbol{\phi}_{j-q}^{\alpha}\right]_{\mu_{0}} a_{j}+\boldsymbol{c}_{\mu_{0}}^{\alpha} a_{2 q+1} .
$$

Note that the sensitivity of the centring is solenoidal with zero values on the boundary $\Gamma_{D}$ according to the Dirichlet boundary condition for the sensitivity problem (see $\left.\S 3.1\right)$. Thus, (2.18) is still valid when using $\boldsymbol{c}_{\mu_{0}}^{\alpha}$ as a test function in the Galerkin method. 


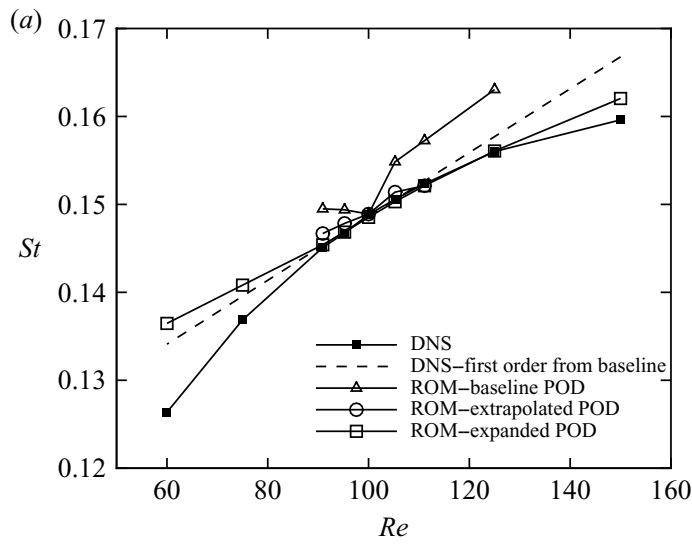

Strouhal number

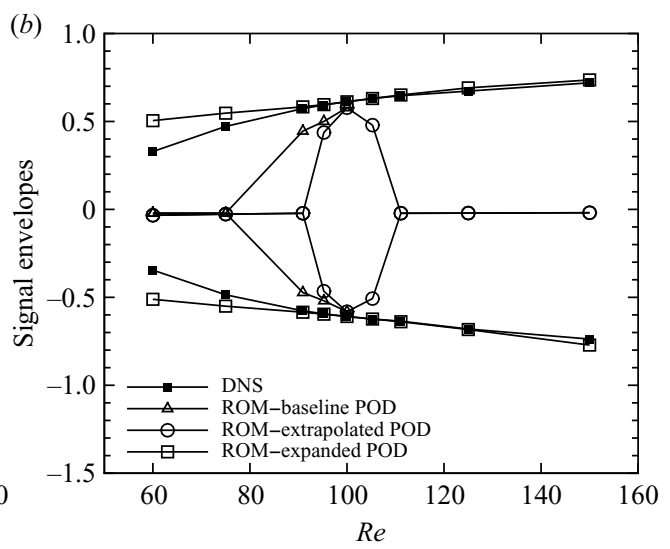

Signal envelopes

FIGURE 15. Reynolds number dependence of ROM for $q=12$.

For the baseline and expanded approaches, a change in the Reynolds number only changes the viscosity coefficient in the low-dimensional system of equations. For the extrapolated approach, the basis and centring are also modified.

We look at the long-term dynamics of the modelled flow response by integrating the low-dimensional systems over 1000 shedding cycles for $q=12$ (the dimension of the system for the expanded approach is thus 25). Their predictions of the Strouhal number for several values of the Reynolds number in the range 60-150 are compared to the full-order Navier-Stokes simulation in figure 15(a). Note that for all states and all approaches, approximated solutions have reached their attractors after this extended integration time. The first-order Taylor series of the DNS is also shown in this figure for comparison

$$
S t(R e)=S t\left(R e_{0}\right)+\frac{\partial S t}{\partial R e}\left(R e-R e_{0}\right) \quad \text { with } \quad \frac{\partial S t}{\partial R e}=-\frac{\mu}{R e} \frac{\partial S t}{\partial \mu},
$$

where the sensitivity of the Strouhal number is evaluated from the rate of growth of the amplitude of the sensitivity of the lift coefficient (see figure 8). Thus, it only uses DNS flow and sensitivity data at the baseline. Note that the complete first-order extrapolation of the flow from the sensitivity solution is only accurate for short integration time and rapidly deteriorates subsequently indicating the need for secondorder Taylor series (see Ilinca et al. 2008). However, the sensitivity frequency can be accurately predicted. Figure $15(b)$ shows the envelopes of the normal component of the flow signals at a location in the cylinder wake $(x=3.152$ and $y=0.064)$.

As can be seen in figure 15(a), the classical baseline approach gives poor prediction of the correct vortex shedding for Reynolds number other than the one for which the model has been built. The model predicts no change in the Strouhal number when reducing the Reynolds number. Furthermore, the amplitude of the signal decreases much too quickly compared to DNS data and reaches a steady-state solution for $R e \leqslant 75$. At these parameter values the solutions of the baseline dynamical systems are attracted to their stable fixed point which is approximately, though not exactly, the mean flow centring. Thus, the frequency of the signal is zero (note that zero frequencies are not shown in figure 15(a) for scaling reasons). This result has already been observed in the literature (cf. Noack et al. 2003). When the Reynolds number increases (i.e. the viscosity coefficient is reduced), the opposite effect is observed and 
(a)

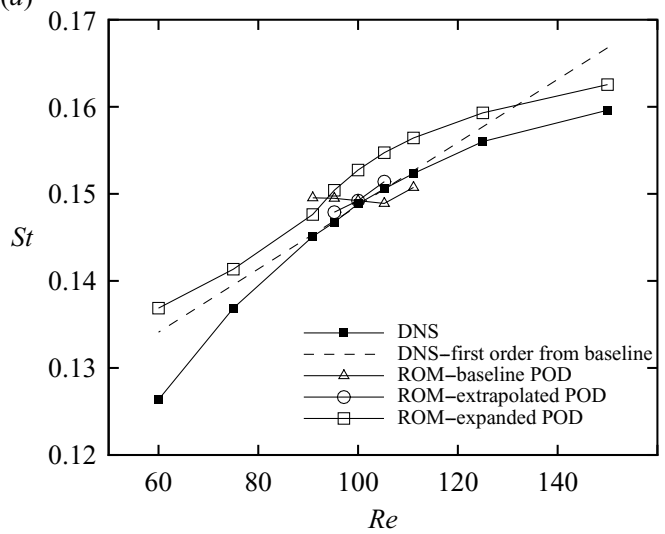

Strouhal number (b)

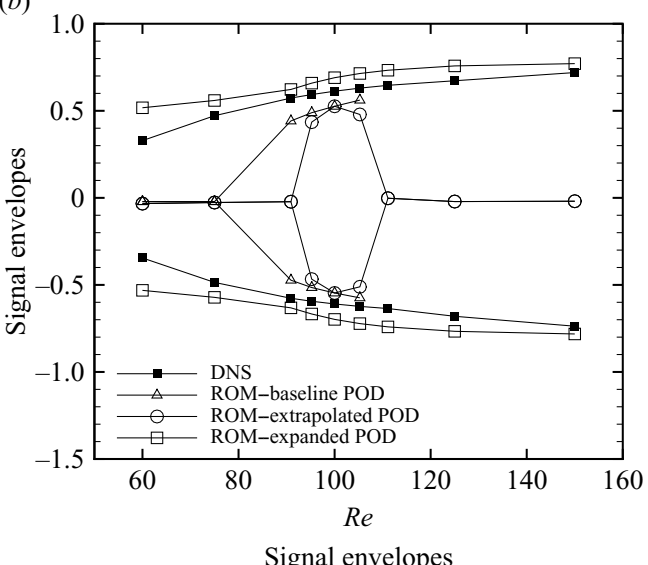

FIGURE 16. Reynolds number dependence of ROM for $q=6$.

the approximated flow response grows rapidly with $R e$. A periodic limit cycle is still reached (except for $R e=150$ where the approximated solution exhibits chaotic fluctuations) but the amplitude of the signal is two orders of magnitude larger than for DNS data (again, these signal envelopes are not plotted in figure $15 b$ for scaling reasons). We insist that these poor predictions of the baseline approach are not due to the truncation in the basis but to inappropriate global spatial functions in the model.

The extrapolated approach provides few improvements since a steady state is reached for $R e \leqslant 75$ and $R e \geqslant 111$. The transient of the solutions simply shows a decrease of the amplitude in time and converges to the final steady solutions. This transient requires a long time to be reached. Again, these results show that this approach is only valid locally close to the baseline $(75 \leqslant R e \leqslant 111)$. In this part of the parameter space, the predictions of the Strouhal number are accurate.

However, the expanded approach approximates the full-order flow responses much better, bringing essentially a linear improvement in the predictions with the Reynolds number (that can be compared to direct first-order extrapolation of the DNS data in the parameter space for the Strouhal number, dashed line in figure 15a). The expanded approach clearly leads to the best results especially for large parameter perturbations. As for DNS data, the attractor of the expanded dynamical system is always a periodic limit cycle. In all cases, it takes less than 50 periods to reach it. Furthermore, these limit cycles are very close to the corresponding full-order limit cycles both in amplitude and frequency. Obviously, the closer to the baseline, the better the accuracy. Yet the improvements of the results is observed over the entire parameter range considered here. The price to pay for these improvements is the doubling of the dynamical system dimension. Better predictions are obtained when the Reynolds number is increased since the flow dependency on this parameter is clearly more linear on this side of the parameter range.

In figure 16, similar behaviour is observed for $q=6$ models constructed using either the baseline or expanded basis approaches. However, even with this low-dimensional system, the expanded approach still reproduces the trend in the flow response with changing Reynolds number and provides a dynamical system that has periodic limit cycles for all the parameter range. Note that at the baseline value $R e=100$, the longterm dynamics of the system show some discrepancies with the baseline approach 


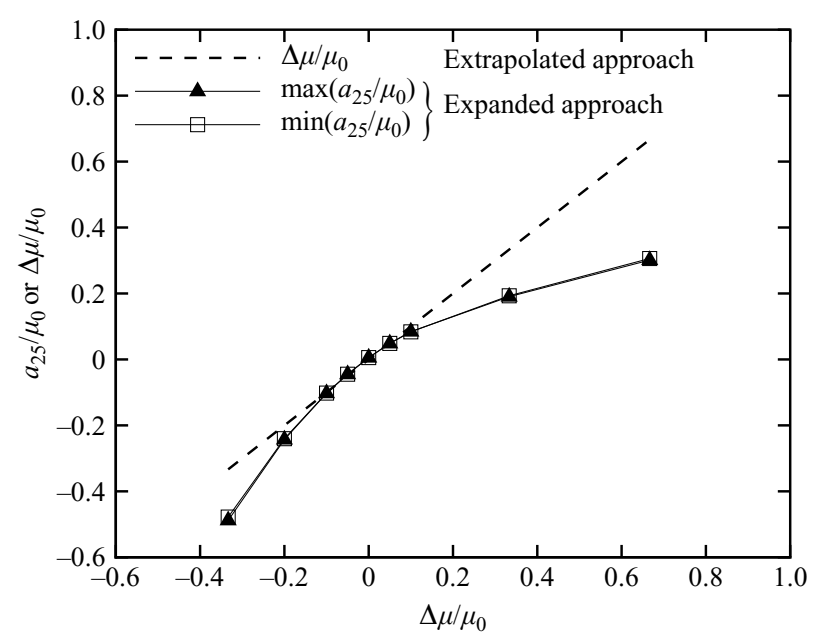

FIGURE 17. Time signature of the sensitivity of the centring with parameter perturbations.

although the sensitivity components (modes and centring) should not be active. Similar results apply for the short-term dynamics in $\S 4.2$. The extrapolated approach does not exhibit such a behaviour since it converges to the baseline approach when the parameter perturbation tends to zero. Due to a very sharp truncation in the spectrum, the baseline approach model does not exactly reproduce the DNS data in figure $16(b)$.

To shed some light on the difference between the extrapolated and expanded approaches, we compare the contributions of the baseline sensitivity of the centring in the approximations (4.4) and (4.5). For the extrapolated approach, the weight of $\boldsymbol{c}_{\mu_{0}}^{\alpha}$ in (4.4) is the parameter perturbation $\Delta \mu$ for all times. For the expanded approach, the weight of $\boldsymbol{c}_{\mu_{0}}^{\alpha}$ is the time coefficient $a_{2 q+1}=a_{25}$ for $q=12$ that is computed by integration of the dynamical system built from the approximation (4.5). Once the stable limit cycle of the expanded dynamical system is reached, we compare the extreme values of $a_{25}$ and the parameter perturbation $\Delta \mu$ over the parameter range in figure 17 (all quantities are normalised by $\mu_{0}$ ). As can be seen, the time coefficient of the sensitivity of the centring in the expanded model is almost constant in time since the differences between its extreme values are negligible. Now, in the vicinity of the baseline, $a_{25}$ values track the parameter perturbation $\Delta \mu$, indicating that the dependency of the centring with the parameter is linear. In this range, the rationale behind the extrapolated approach is validated. In this linear range, enlarging the basis by including the centring sensitivity is unnecessary. This motivates the choice of the extrapolated basis in this parameter range. However, for larger parameter changes the departure from the linearity becomes more and more pronounced. In this case, the expanded approach offers more flexibility in defining the weight of $\boldsymbol{c}_{\mu_{0}}^{\alpha}$ in the reduced-order solution and leads to better models. Though such a simple analysis is not possible for the sensitivity modes, this phenomena may help explain the better behaviour of the expanded basis away from the baseline.

We now look at the long-term behaviour (last 10 shedding periods) of the dynamical systems in the phase planes $a_{1}-a_{2}$ and $a_{1}-a_{3}$ for the baseline and expanded approaches in figures 18 and 19 for $R e=111.11\left(\Delta \mu / \mu_{0}=-10 \%\right)$ and $R e=150\left(\Delta \mu / \mu_{0}=\right.$ $-33 \%$ ), respectively (again for $q=12$ ). In these figures, the solid lines are the predictions of the low-dimensional models and the dashed lines are the projection of 

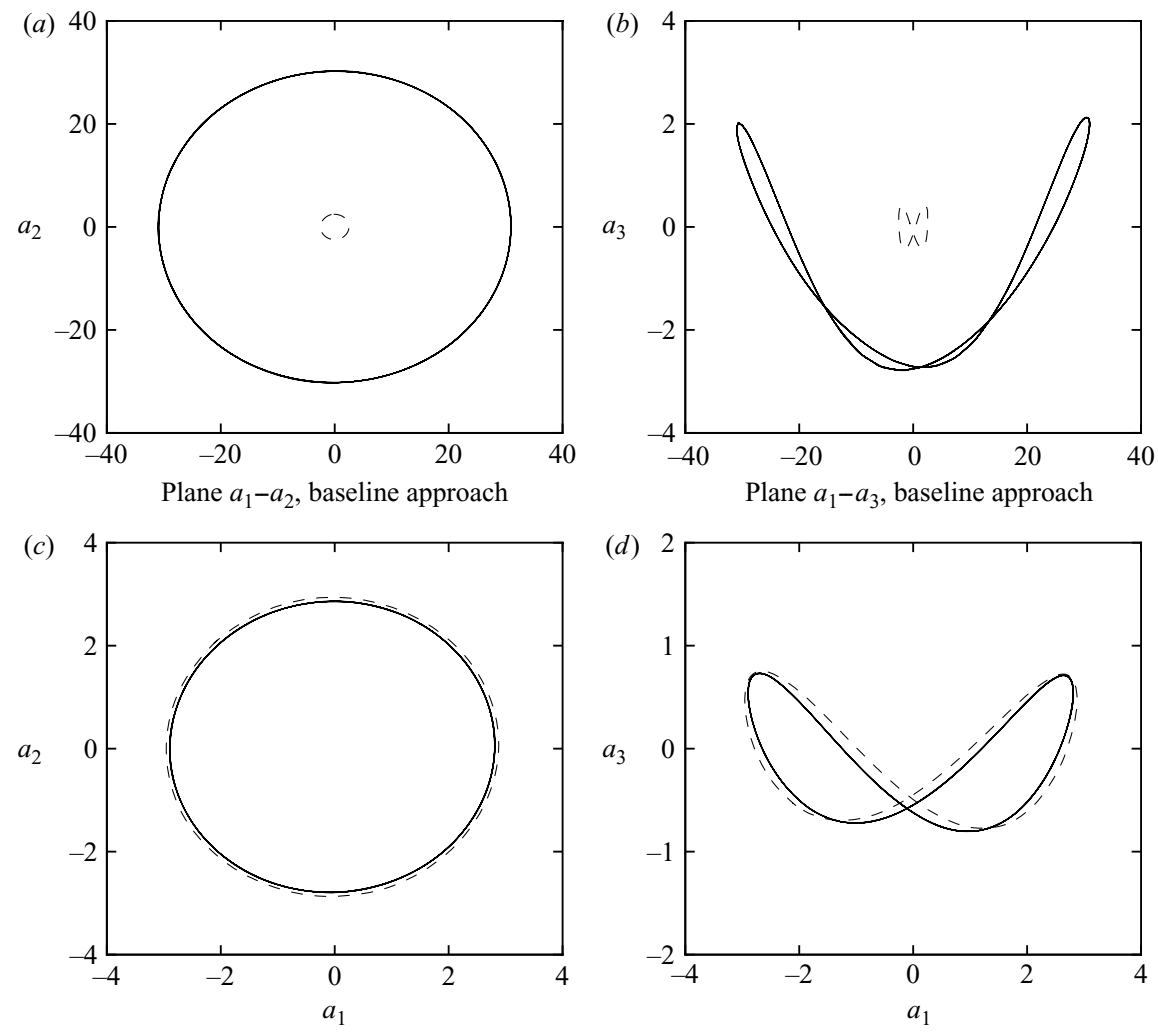

Plane $a_{1}-a_{2}$, expanded approach

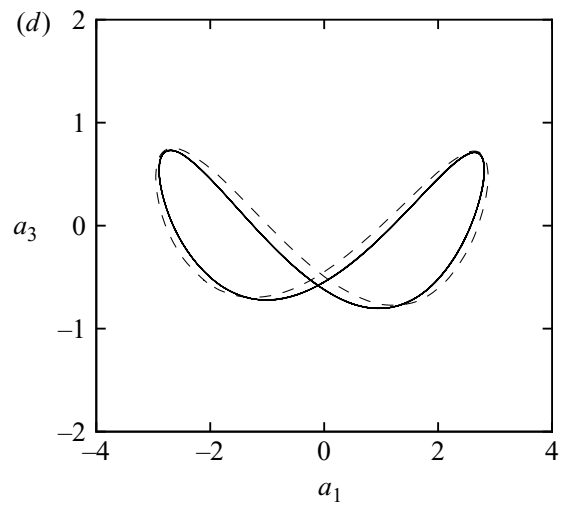

Plane $a_{1}-a_{3}$, expanded approach

FIGURE 18. $R e=111.11, q=12$ (solid lines: ROM, dashed lines: DNS).

the modes on the full-order simulation at the corresponding states. As can be seen, the baseline approach brings very poor predictions. For $R e \approx 111$, a stable limit cycle is reached but it is far from the attractor of the full-order simulation. For $R e \approx 150$, the shape of the attractors are significantly different. However, the attractor of the dynamical system built from the expanded approach approximates the full-order one fairly well even for the largest parameter perturbation.

For the sake of completeness, figure 20 shows the same results for the expanded approach with $q=6$. With fewer components, the model is not as accurate but the attractor of the low-dimensional system has still the same qualitative shape as the full-order attractor.

\section{Conclusion}

This paper studies the robustness of low-dimensional models for fluid flows. These models are obtained by the Galerkin projection of the Navier-Stokes equations onto a reduced basis. The proper orthogonal decomposition is the prevailing method to generate low-dimensional bases. It has an interpretation of providing optimal reduced bases in terms of kinetic energy representation. Since it requires computationally demanding DNS data, the basis generation is the most expensive step in building reduced-order models. The classical approach is to build the basis for a particular set of the parameters defining the problem at hand. However, the resulting reduced basis is only optimal at these particular states and may perform poorly in representing 

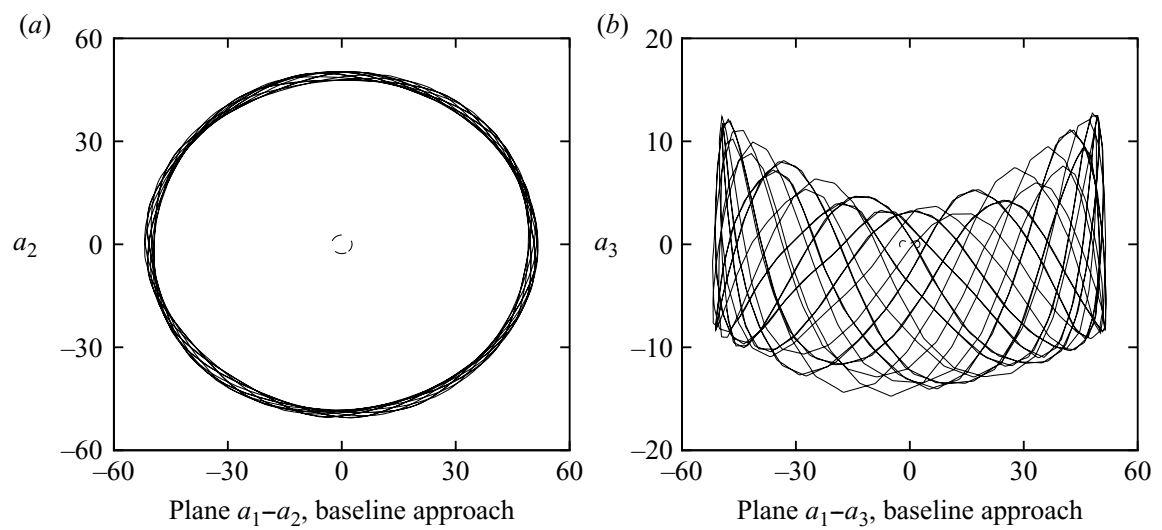

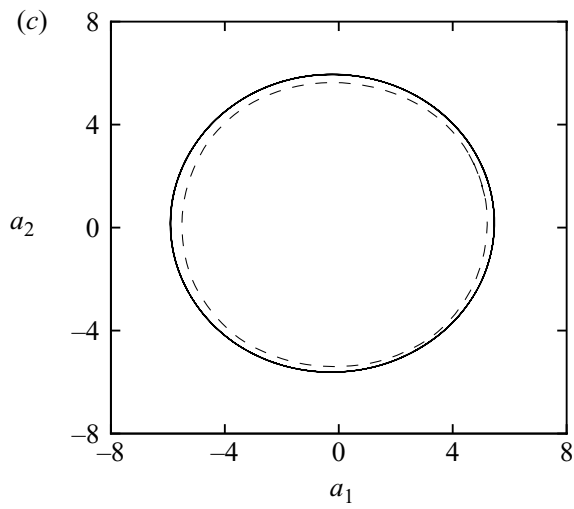

Plane $a_{1}-a_{2}$, expanded approach

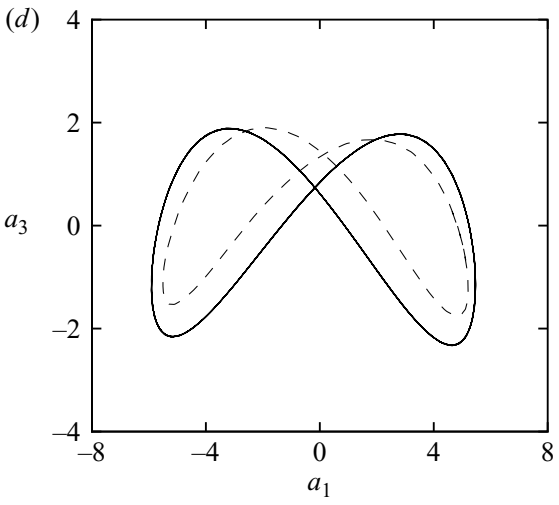

Plane $a_{1}-a_{3}$, expanded approach

Figure 19. $R e=150, q=12$ (solid lines: ROM, dashed lines: DNS).

flow solutions for different sets of parameters. This drawback was reported in the literature for laminar flows over obstacles for a range of Reynolds numbers.

To partially circumvent this issue, we have presented a method for computing the sensitivity of the POD. It consists of differentiating the eigenvalue problem corresponding to the POD with respect to the parameters and involves the sensitivity of the DNS data. The latter has been obtained by the sensitivity equation method and requires the solution of a set of PDEs for the sensitivity of the flow variables. The main advantage of this approach is that the sensitivity of the data are obtained for a fraction of the cost of the flow data since the sensitivity equations are always linear. The solution of the differentiated eigenvalue problem provides both the sensitivity of the eigenvalues and the POD modes. They first provide information on how these temporal and spatial modes vary with changes in the parameter. This information is valuable because the POD is not only used for model building purposes but also for flow characterization. For the flows over a square cylinder, the sensitivity of the eigenvalues with respect to the fluid viscosity are almost all negative, indicating that an increase in this parameter will lead to a reduction of the total kinetic energy of the flow. This is consistent with our understanding of these well-known flows. Secondly, the POD mode sensitivities can be used in the basis selection process to enlarge the set of solutions that can be accurately represented in the parameter space. To this end, we have examined two different approaches. The first one extrapolates the POD modes in the parameter space to the corresponding state to be modelled. It relies on the 
(a)

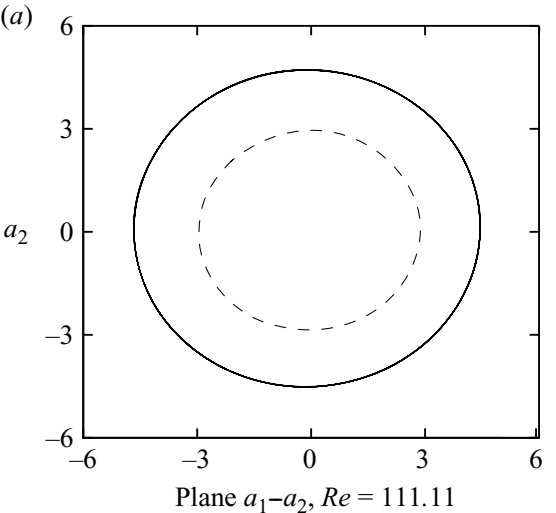

(c)

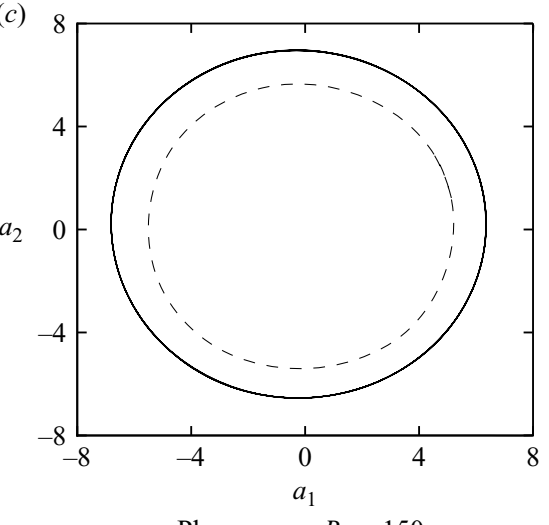

(b)

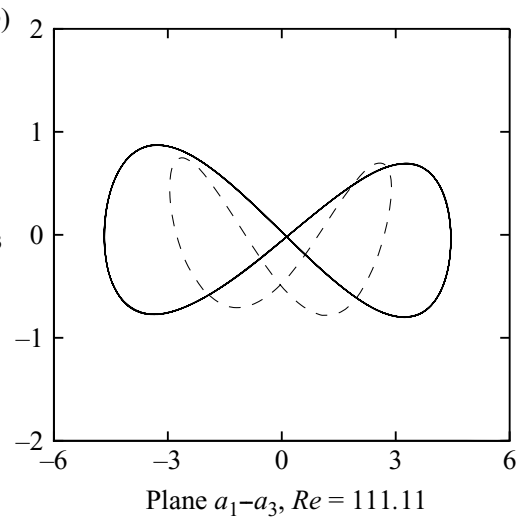

(d)

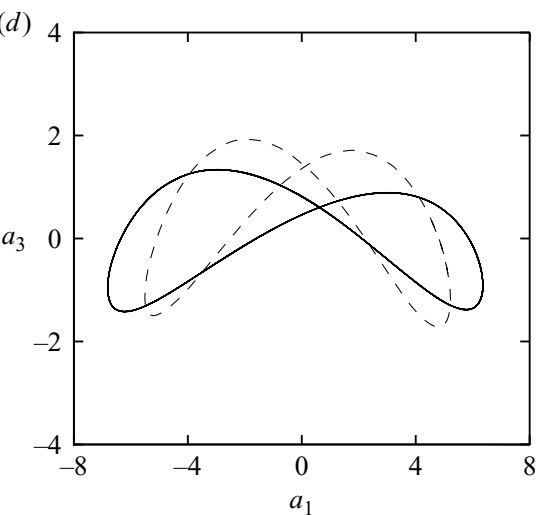

Plane $a_{1}-a_{3}, R e=150$

FIGURE 20. Expanded approach, $q=6$ (solid lines: ROM, dashed lines: DNS).

assumption that these eigenfunctions have a linear dependency on the parameters that can only be validated locally. The second one expands the reduced basis by adding the sensitivity of the original modes to the original POD basis. The underlying idea behind this approach is that the POD mode sensitivities span a different subspace than those generated by the POD eigenfunctions and point in relevant directions when considering parameter changes. This property has been demonstrated for the case of flows over a square cylinder.

The new bases have each led to an improvement in representing the data for a range of Reynolds numbers. However, the extrapolation approach offers only limited improvements very locally around the baseline. For larger variations in the parameter space, it quickly loses accuracy. Clearly, the expanded approach provides a better and more robust alternative but essentially doubles the basis dimension. The short-term solution of the resulting ROMs approximates the DNS data fairly well given the inclusion of sufficient components in the basis. Using only data extracted at a single Reynolds number (initial conditions, mean flow, modes and their sensitivities), the long-term dynamics of the low-dimensional model is in good agreement with the DNS data both in terms of amplitude and frequency. It has been shown that the attractors of the ROMs are close to the full-order attractor. However, the expansion also constitutes a linear approach in essence and loses accuracy for the largest parameter 
perturbations examined in this study. Thus, the efficiency of this approach depends on how linear the flow dynamics are with respect to parameter changes.

Our future research will aim at extending these ideas to shape parameters with the aim of producing reduced-order models for parameter-dependent geometries.

This research was supported in part by the Air Force Office of Scientific Research (under contract FA9550-08-1-0136), the National Science Foundation (under contract DMS-0513542), the National Science and Engineering Council of Canada and the Canadian Research Chair Program.

\section{REFERENCES}

Antoulas, A. C. 2005 Approximation of Large-Scale Dynamical Systems. Advances in Design and Control. SIAM.

Antoulas, A. C., Sorensen, D. C. \& Gugercin, S. 2001 A survey of model reduction methods for large-scale systems. Contemp. Math. 280, 193-219.

Aubry, N., Holmes, P., Lumley, J. L. \& Stone, E. 1988 The dynamics of coherent structures in the wall region of a turbulent boundary layer. J. Fluid Mech. 192, 115-173.

Bangia, A. K., Batcho, P. F., Kevrekidis, I. G. \& Karniadakis, G. E. 1997 Unsteady twodimensional flows in complex geometries: comparative bifurcation studies with global eigenfunction expansions. SIAM J. Sci. Comput. 18, 775-805.

Bergmann, M., Cordier, L. \& Brancher, J.-P. 2005 Optimal rotary control of the cylinder wake using proper orthogonal decomposition reduced-order model. Phys. Fluids 17 (9), 097101:121.

BorggaARd, J. \& BURns, J. 1997 A PDE sensitivity equation method for optimal aerodynamic design. J. Comput. Phys. 136 (2), 367-384.

Borggaard, J., Hay, A. \& Pelletier, D. 2007 Interval-based reduced-order models for unsteady fluid flow. Intl J. Numer. Anal. Model. 4 (3-4), 353-367.

Couplet, M., Basdevant, C. \& Sagaut, P. 2005 Calibrated reduced-order POD-Galerkin system for fluid flow modeling. J. Comput. Phys. 207 (1), 192-220.

Davis, R. W. \& Moore, E. F. 1982 A numerical study of vortex shedding from rectangles. J. Fluid Mech. 116, 475-506.

Deane, E. A., Kevrekidis, I. G., Karniadakis, G. E. \& Orszag, S. A. 1991 Low-dimensional models for complex geometry flows: application to grooved channels and circular cylinders. Phys. Fluids A 3 (10), 2337-2354.

FAHL, M. 2000 Trust-region methods for flow control based on reduced order modelling. PhD thesis, Universität Trier, Germany.

Fox, R. L. \& KAPOOR, M. P. 1968 Rates of change of eigenvalues and eigenvectors. AIAA J. 6 (12), $2426-2429$.

Franke, R., Rodi, W. \& Schoaconung, B. 1990 Numerical calculation of laminar vortex-shedding flow past cylinders. J. Wind Engng Ind. Aerodyn. 35, 237-257.

Galletti, B., Bruneau, C. H., Zannetti, L. \& Iollo, A. 2004 Low-order modelling of laminar flow regimes past a confined square cylinder. J. Fluid Mech. 503, 161-170.

Ganapathysubramanian, S. \& Zabaras, N. 2004 Design across length scales: a reduced-order model of polycrystal plasticity for the control of microstructure-sensitive material properties. Comput. Meth. Appl. Mech. Engng 193, 5017-5034.

Graham, W. R., Peraire, J. \& Tang, K. T. $1999 a$ Optimal control of vortex shedding using low order models. Part 1. Open-loop model development. Intl J. Numer. Methods Engng 44 (7), 945-972.

Graham, W. R., Peraire, J. \& Tang, K. T. $1999 b$ Optimal control of vortex shedding using low order models. Part 2. Model-based control. Intl J. Numer. Methods Engng 44 (7), 973-990.

Hay, A., Borggand, J. \& Pelletier, D. 2008 On the use of sensitivity analysis to improve reducedorder models. In 4th AIAA Flow Control Conference, Seattle, Washington, AIAA-2008-4192.

Holmes, P., Lumley, J. L. \& Berkooz, G. 1996 Turbulence, Coherent Structures, Dynamical Systems and Symmetry. Cambridge University. 
Hotelling, H. 1933 Analysis of a complex of statistical variables with principal components. J. Educat. Psych. 24, 417-441.

Hristova, H., Etienne, S., Pelletier, D. \& Borggaard, J. 2006 A continuous sensitivity equation method for time-dependent incompressible laminar flows. Intl J. Numer. Meth. Fluids $\mathbf{5 0}$ (7), $817-844$.

Ilinca, F., Pelletier, D. \& Hay, A. 2008 First- and second-order sensitivity equation methods for value and shape parameters. Intl J. Numer. Meth. Fluids 57 (9), 1349-1370.

Ito, K. \& RaVInDRAN, S. 1996 Reduced basis method for flow control. Tech Rep. CRSC-TR96-25, Center for Research in Scientific Computation, North Carolina State University, Raleigh, NC.

Karhunen, K. 1946 Zur Spektraltheorie stochastischer Prozesse. Annales Academiae Scientiarum Fennicae 37.

KunisCH, K. \& VolKweIN, S. 1999 Control of Burgers' equation by a reduced order approach using proper orthogonal decomposition. J. Optim. Theor. Appl. 102, 345-371.

Kunisch, K., VolKweIn, S. \& XIE, L. 2004 HJB-POD-based feedback design for the optimal control of evolution problems. SIAM J. Appl. Dyn. Syst. 3 (4), 701-722.

LANCASTER, P. 1964 On eigenvalues of matrices dependent on a parameter. Numer. Math. 6, 377387.

Lehmann, O., Luchtenburg, D. M., Noack, B. R., King, R., Morzyński, M. \& Tadmor, G. 2005 Wake stabilization using POD Galerkin models with interpolated modes. In 44th IEEE Conference on Decision and Control (CDC) and European Control Conference (ECC), Sevilla, Spain.

Lieu, T., Farhat, C. \& Lesoinne, M. 2006 Reduced-order fluid/structure modeling of a complete aircraft configuration. Comput. Meth. Appl. Mech. Engng 195, 5730-5742.

LoÈve, M. 1955 Probability Theory. Van Nostrand.

LoRENZ, E. N. 1956 Empirical orthogonal functions and statistical weather prediction. Tech Rep. M.I.T.

Ma, X. \& Karniadakis, G. E. 2002 A low-dimensional model for simulating three-dimensional cylinder flow. J. Fluid Mech. 458, 181-190.

Morzyński, M., Afanasiev, K. \& Thiele, F. 1999 Solution of the eigenvalue problems resulting from global non-parallel flow stability analysis. Comput. Meth. Appl. Mech. Engng 169, 161-176.

Morzyński, M., Stankiewicz, W., Noack, B. R., King, R., Thiele, F. \& Tadmor, G. 2007 Continuous mode interpolation for control-oriented models of fluid flows. In Active Flow Control (ed. R. King), pp. 260-278. Springer-Verlag.

Murthy, D. V. \& HaftKa, R. T. 1988 Derivatives of eigenvalues and eigenvectors of a general complex matrix. Intl J. Numer. Meth. Engng 26, 293-311.

Noack, B. R., Afanasiev, K., Morzyński, M., Tadmor, G. \& Thiele, F. 2003 A hierarchy of low-dimensional models for the transient and post-transient cylinder wake. J. Fluid Mech. 497, 335-363.

NoAcK, B. R. \& EcKelmann, H. 1994a A global stability analysis of the steady and periodic cylinder wake. J. Fluid Mech. 270, 297-330.

NoAcK, B. R. \& ECKelmann, H. $1994 b$ A low-dimensional Galerkin method for the threedimensional flow around a ciruclar cylinder. Phys. Fluids 6, 124-143.

Okajima, A. 1982 Strouhal number of rectangular cylinders. J. Fluid Mech. 123, 379-398.

Pelletier, D., Hay, A., Etienne, S. \& BorggaArd, J. 2008 The sensitivity equation method in fluid mechanics. Eur. J. Comput. Mech. 17 (1-2), 31-61.

Peterson, J. S. 1989 The reduced basis method for incompressible viscous flow calculations. SIAM J. Sci. Stat. Comput. 10 (4), 777-786.

Rowley, C. W. \& Williams, D. R. 2006 Dynamics and control of high-Reynolds-number flow over open cavities. Annu. Rev. Fluid Mech. 38, 251-276.

Saha, A. K., Biswas, G. \& Muralidhar, K. 2003 Three-dimensional study of flow past a square cylinder at low Reynolds numbers. Intl J. Heat Fluid Flow 24, 54-66.

Seyranian, A. P., Lund, E. \& Olhoff, N. 1994 Multiple eigenvalues in structural optimization problems. Struct. Optim. 8, 207-227.

Sirisup, S. \& Karniadakis, G. E. 2004 A spectral viscosity method for correcting the long-term behavior of POD models. J. Comput. Phys. 194, 92-116.

Sirovich, L. 1987 Turbulence and the dynamics of coherent structures. Part I. Coherent structures. Q. Appl. Math. 45 (3), 561-571. 
Sohankar, A., Norberg, C. \& Davidson, L. 1997 Numerical simulation of unsteady low-Reynolds number flow around rectangular cylinders at incidence. J. Wind Engng Ind. Aerodyn. 69-71, 189-201.

Sohankar, A., Norberg, C. \& Davidson, L. 1998 Low-Reynolds-number flow around a square cylinder at incidence: study of blockage, onset of vortex shedding and outlet boundary condition. Intl J. Numer. Meth. Fluids 26 (1), 39-56.

Sohankar, A., Norberg, C. \& Davidson, L. 1999 Simulation of three-dimensional flow around a square cylinder at moderate Reynolds numbers. Phys. Fluids 11 (2), 189-201. 